\title{
Article \\ LDM-Ex-FDM: A Novel Multi-Service Transmission Scheme for the ATSC 3.0 System
}

\author{
Xianzheng Deng ${ }^{1,2}$, Xin Bian ${ }^{1}$ and Mingqi $\mathrm{Li}^{1, * \mathbb{C}}$ \\ 1 Shanghai Advanced Research Institute, Chinese Academy of Sciences, Shanghai 201210, China; \\ dengxianzheng@sari.ac.cn (X.D.); bianx@sari.ac.cn (X.B.) \\ 2 University of Chinese Academy of Sciences, Beijing 100049, China \\ * Correspondence: limq@sari.ac.cn
}

Citation: Deng, X.; Bian, X.; Li, M. LDM-Ex-FDM: A Novel Multi-Service Transmission Scheme for the ATSC 3.0 System. Appl. Sci. 2021, 11, 3178. https://doi.org/ 10.3390/app11073178

Academic Editor: Christos Bouras

Received: 11 March 2021

Accepted: 1 April 2021

Published: 2 April 2021

Publisher's Note: MDPI stays neutral with regard to jurisdictional claims in published maps and institutional affiliations.

Copyright: (c) 2021 by the authors. Licensee MDPI, Basel, Switzerland. This article is an open access article distributed under the terms and conditions of the Creative Commons Attribution (CC BY) license (https:// creativecommons.org/licenses/by/ $4.0 /)$.

\begin{abstract}
In order to improve system coverage performance, in this paper, a multi-service transmission scheme on the basis of the puncturing technique, namely layered division multiplexing extension frequency-division multiplexing (LDM-Ex-FDM), is proposed. The key idea of the proposed scheme is that the symbols punctured from the enhanced layer $(E L)$ of fixed services will be independently transmitted on a certain number of subcarriers orthogonal to the $L D M$ signal of the core layer $(C L)$ and most of the EL. By doing so, the punctured symbols will be demodulated with a higher signalto-noise ratio (SNR) on the receiver side and can be recovered well, thus improving the reception performance of the EL fixed services. Moreover, two puncturing strategies based on bit-interleaved coded modulation (BICM) symbols of the LDM-Ex-FDM scheme, namely equal interval symbol puncturing (EISP) and non-equal interval symbol puncturing (NEISP), are developed to improve the performance of the EL services. Simulation results are given to show that, when the puncturing rate in the LDM-Ex-FDM scheme is configured as 1/12, the performance of the fixed service can be improved by $1.8 \mathrm{~dB}$, meanwhile the bit error rate (BER) performance of the mobile service is not affected. Thus, the overall system coverage performance can be improved. Furthermore, to reduce the computational complexity and the demodulation delay of fixed service carried in the $E L$, at the receiver, a direct interference cancellation (DIC) detector is proposed as well. Simulation results are given to show that, under high injection level and high modulation order, the proposed DIC scheme is able to achieve almost the same BER performance as that of the traditional successive interference cancellation (SIC) scheme while with lower computational complexity.
\end{abstract}

Keywords: LDM; ATSC 3.0; FDM; Multi-service transmission; SIC

\section{Introduction}

Digital TV programs can be broadcast via terrestrial, satellite, and cable networks, in which the digital terrestrial television broadcasting (DTTB) system is particularly important in the broadcast coverage network. In recent years, the next generation DTTB which supports ultra-high definition TV (UHDTV) has become one of the research hotspots in academia and industry [1]. On the one hand, in addition to the fixed service reception of ultra-high definition programs, with the proliferation of a large number of intelligent portable terminals, the demand for a variety of mobile services is also increasing. On the other hand, the available spectrum resources for DTTB are limited. Therefore, it is of great importance to efficiently use spectrum resources and flexibly meet the growing multiservice requirements in the future. Many terrestrial broadcasting standards have been published internationally. In September 2009, the European Telecommunications Standards Institute (ETSI) released the first version of the DVB-T2 standard [2]. In July 2015, digital terrestrial television multimedia broadcasting-advanced (DTMB-A), China's next-generation digital television broadcasting system was officially adopted by the International Telecommunication Union (ITU) as the international standard [3]. In September 2016, the American 
next-generation terrestrial broadcasting system Advanced Television System Committee (ATSC) 3.0 physical layer standard was released [4]. It is worth pointing out that DVBT2, DTMB-A, and ATSC 3.0 all support 4K UHDTV and are suitable for multi-service transmission of both mobile and fixed services [4-6].

In the ATSC 3.0 standard, the layered division multiplexing ( $L D M)$ technique is used, which is a non-orthogonal multiple access (NOMA) scheme of air interface technologies. Point-to-multipoint (P2MP) transmission mode will be supported in future networks to achieve high spectrum efficiency and high capacity [7]. Eventually, NOMA and P2MP have been identified as key technologies for more efficient use of spectrum in 5G [8-10]. Reference [7] proves that the 5G P2MP subsystem with LDM can make use of the broadband infrastructure to provide high-quality broadcast-type services. $L D M$ enables the simultaneous delivery of different services in the same time-frequency resources. One typical scenario for an LDM-based ATSC 3.0 system is to deliver a robust high-definition TV (HDTV) service in the core layer (CL) for mobile reception and a 4K UHDTV service in the enhanced layer $(E L)$ for fixed reception [11]. One of the technical advantages of $L D M$ is that it can achieve a lower signal-to-noise ratio (SNR) threshold under the same data throughput. Another advantage is that, compared to the traditional time-division multiplexing (TDM) and/or frequency-division multiplexing (FDM), the spectrum efficiency of $L D M$ is much higher [12-14].

So far, there have been extensive studies on the performance improvement of the $E L$ of $L D M$ in typical scenarios in the literature, where the integration of $L D M$ and TDM/FDM is an effective way to improve the performance of the multi-service transmission. Reference [15] proposes a multi-physical layer pipe (M-PLP) configuration scheme called time-layered division multiplexing (TLDM)/ layered-time division multiplexing (LTDM), which proves the advantages of the $L D M$ technology to provide hierarchical broadcasting services. However, if the new service mentioned therein is a truncated part of the $E L$ services, the improvement of the overall performance of the $E L$ in this case would be very limited. Moreover, the capacity of the $C L$ services will be decreased due to the reduced time duration caused by the combination of the TDM. Reference [16] studied the elimination method of the interference of the $C L$ signal to the $E L$, which can improve the performance of the latter. However, the $E L$ and $C L$ of the $L D M$ signal in reference [16] carries the same services in the considered scenario. Reference [17] proposes a low-rate low-density parity-check (LDPC) code applied to CL of $L D M$, aiming to increase the power of EL by reducing the injection level of $L D M$, and finally, the improved reception performance of $E L$ is obtained. However, this approach is only suitable for very robust services delivered using $C L$. Although reference $[17,18]$ mentioned the combination between $L D M$ and FDM, unfortunately, the detailed analysis is absent.

In this paper, we will investigate the transceiver structure and evaluate the performance of the combination between $L D M$ and FDM in a comprehensive manner. The first objective of this work is to develop an efficient transceiver structure with the aid of symbol puncturing techniques, thus improving the performance of the $E L$ services without affecting the reception threshold of the $C L$ services. The key idea of the proposed scheme is that the symbols punctured from the $E L$ of fixed services will be independently transmitted on a certain number of subcarriers orthogonal to the $L D M$ signal of the $C L$ and most of the EL. Then, the punctured symbols will be demodulated with a higher SNR on the receiver side and can be recovered well, thus improving the reception performance of the EL fixed services.

For the demodulation of $L D M$ signals, the existing detection methods usually use a successive interference cancellation (SIC) detector to perform multi-level signal detection at the receiver. However, for a receiving terminal that only demodulates the EL signal of the $L D M$, if it is forced to demodulate the $C L$ signal of the $L D M$ first, and then performs the bit-interleaved coded modulation (BICM) signal reconstruction on it, additional complexity and signal processing delay will inevitably increase. In view of the increase in complexity at the transmitter side as a result of the introduction of symbol puncturing, the second 
objective of this work is to further decrease the computational complexity at the receiver side. Fortunately, note that $L D M$ can be regarded as the extension of low-order modulation to high-order modulation during EL subcarrier modulation. Compared with the singlelayer signal structure, the constellation after the $E L$ symbol mapping has a constellation offset. Therefore, correcting the constellation offset of the EL symbol is equivalent to eliminating the interference of the $C L$ to the $E L$, so that the $E L$ signal can be demodulated. Motivated by that, a direct interference cancellation (DIC) detector is also developed in this paper, where the fixed receiver can decode the $E L$ symbol just by correcting the constellation offset of the EL symbol on the received LDM signal.

In summary, the novel contributions of this paper are as follows:

- We propose a novel combined FDM/LDM multi-service transmission scheme, namely LDM-Ex-FDM, which is used to improve the reception performance of $E L$ services without degrading the SNR threshold of $C L$ services, thus enhancing the system coverage performance.

- Two BICM symbol puncturing strategies of the LDM-Ex-FDM scheme are proposed, i.e., equal interval symbol puncturing (EISP) and non-equal interval symbol puncturing (NEISP). The LDM-Ex-FDM scheme with EISP can be used for multi-service transmission under typical scenarios while the LDM-Ex-FDM scheme with NEISP performs better in multi-service transmission scenarios with low power injection levels, which will bring about the serious overlap of the constellation symbols of the LDM composite signal.

- At the receiving end, a DIC detector is also proposed to reduce the computational complexity and the signal processing delay. The effectiveness of the proposed detector is verified by simulation.

The remainder of this paper is briefly described as follows. Section 2 gives an overview of the ATSC 3.0 system. Section 3 introduces the transceiver structure of the LDM-Ex-FDM multi-service transmission scheme for the ATSC 3.0 system. The BICM capacity of the proposed scheme will also be analyzed in Section 3. In Section 4, simulation results and discussions will be given to evaluate the system performance of the proposed LDM-ExFDM scheme. Finally, Section 5 draws the conclusion.

\section{Advanced Television System Committee (ATSC) 3.0 System Overview}

Figure 1 shows the basic transceiver structure of $L D M$ in the ATSC 3.0 system. To highlight the core idea, additional information such as signaling and pre-synchronization are not shown in this figure. At the transmitter, the ATSC 3.0 system provides many different configuration modes according to different requirements for robustness and capacity by different services. Besides, every layer can share the same orthogonal frequency division multiplexing (OFDM) numerologies, including fast Fourier transform (FFT) size, pilot structure, and cyclic prefix $(\mathrm{CP})$ etc. From the perspective of low implementation complexity, only a two-layer LDM scheme is adopted in the ATSC 3.0 physical layer standard where the transmission power can be allocated to the two layers by adjusting the power injection level. In a typical two-layer $L D M$ system, $C L$ signals usually use a more robust coding and modulation configuration than EL signals.

At the receiver, the optimal maximum likelihood (ML) detection can be employed to detect the signals of all layers. However, the complexity of the ML detector can be extremely high. The existing detection scheme of two-layer LDM is based on the SIC detector, as illustrated in Figure 1b. Firstly, this part demodulates the most robust $C L$ signal during which the EL signal is disposed as noise. Secondly, it decodes the $C L$ signal to obtain the bit information for mobile services. Thirdly, it carries out BICM reconstruction of the bit information to generate $C L$ symbols, which are then subtracted from the received $L D M$ signal. Finally, obtains the bit data for fixed service by decoding the EL. 


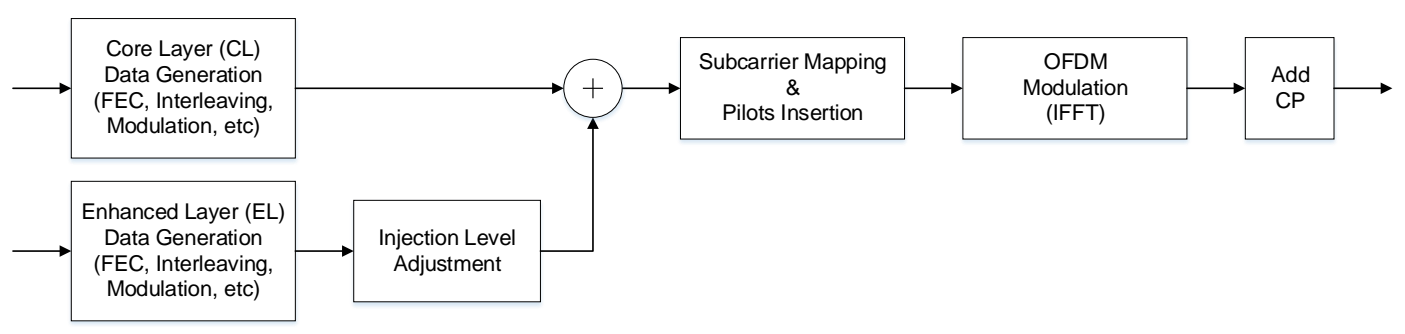

(a) ATSC 3.0 LDM Transmitter

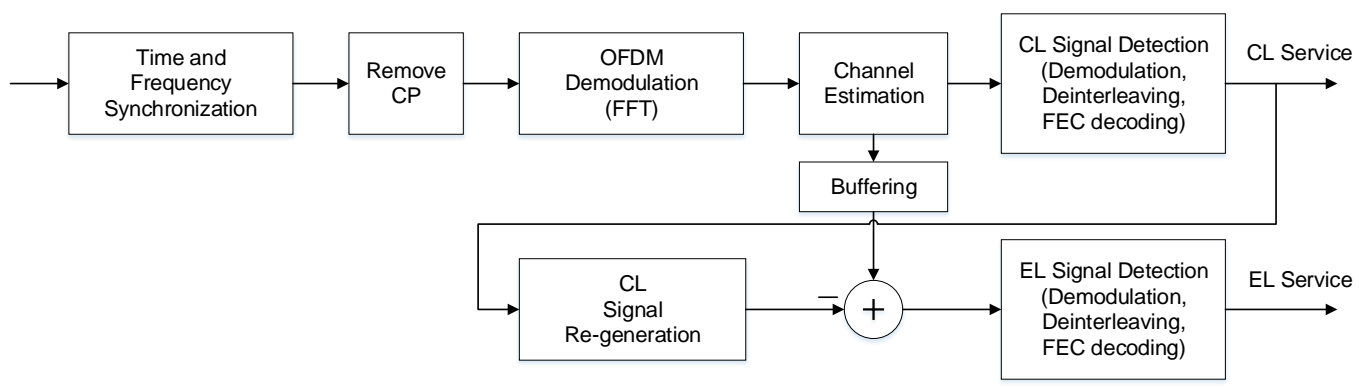

(b) ATSC 3.0 LDM Receiver

Figure 1. The two-layer layered division multiplexing ( $L D M)$ transmitter and receiver of the traditional Advanced Television System Committee (ATSC) 3.0 system: (a) two-layer LDM transmitter; (b) two-layer LDM receiver.

\section{Layered Division Multiplexing Extension Frequency-Division Multiplexing (LDM-Ex-FDM) Scheme for the ATSC 3.0 System}

The traditional $L D M$ scheme cannot improve the performance of $E L$ while maintaining the bit error rate (BER) performance of $C L$. In this section, the reasons why the proposed LDM-Ex-FDM scheme can solve this problem will be explained, and the LDM-Ex-FDM multi-service transmission scheme and the low-complexity DIC scheme for fixed service reception will be described in detail.

\subsection{System Model}

In the typical scenario of using LDM in the ATSC 3.0 system, simply adjusting the injection level can improve the reception performance of the high throughput fixed service carried in the $E L$ of the $L D M$. However, it will significantly affect the reception of the mobile service of the $C L$. Therefore, how to improve the reception performance of the $E L$ without affecting the system performance of the $C L$ is meaningful. This paper attempts to tackle this problem by resorting to the combination of $L D M$ with FDM.

Specifically, the data symbols for the mobile service stream are transmitted through the $C L$ of $L D M$ while the data symbols for fixed service stream are divided into two parts: $E L 1$ and EL2. EL1 is assigned to the lower layer of $L D M$. The $L D M$ signal formed by the multiplexing of $E L 1$ and $C L$ is frequency division multiplexed with $E L 2$, which then forms the final composite frame structure. In this case, the SNR of EL1 is much lower than that of EL2. To perform the error correction of the BICM more effectively, puncturing at equal intervals is adopted when dividing the data symbols of the fixed service stream. The remaining symbols after puncturing are $E L 1$ and the symbols removed by puncturing are $E L 2$. The amount of information carried by EL1 is larger than that by EL2.

According to different service requirements, the information to be transmitted is formed into modulated symbols of different layers through different forward error-correction (FEC) coding and the BICM process, and then the modulated symbols are mapped to OFDM subcarriers. The transmitted symbols of the $L D M$ signal can be expressed as:

$$
X_{L D M}(k)=\sqrt{\frac{1}{1+\alpha}} X_{C L}(k)+\sqrt{\frac{\alpha}{1+\alpha}} X_{E L 1}(k)
$$




$$
\Delta=10 \cdot \lg \frac{1}{\alpha}
$$

where $k$ represents the subcarrier index, $\alpha$ denotes the average power ratio of the $L D M$, $\Delta$ indicates the injection level. $X_{C L}(k)$ and $X_{E L 1}(k)$ stand for the transmitted symbols for $C L$ reception and $E L$ reception on the $k$-th sub-carrier, respectively. Note that the average power of $X_{C L}(k)+X_{E L 1}(k)$ is normalized. Through a time-invariant channel, at the receiver, the received symbols can be denoted as:

$$
\begin{aligned}
Y_{L D M}(k) & =\sqrt{\frac{1}{1+\alpha}} X_{C L}(k) \cdot H(k) \\
& +\sqrt{\frac{\alpha}{1+\alpha}} X_{E L 1}(k) \cdot H(k)+N(k)
\end{aligned}
$$

where $H(k)$ represents the transfer function, $N(k)$ stands for the Gaussian noise and other additive interference. After channel estimation, the $C L$ symbols can be demodulated through the following single-tap frequency-domain (FD) equalization, i.e.,

$$
\begin{aligned}
\hat{X}_{C L}(k) & =\frac{\gamma_{L D M}(k) \sqrt{1+\alpha}}{\hat{H}(k)} \\
& =X_{C L}(k) \frac{H(k)}{\hat{H}(k)} \\
& +\frac{\sqrt{\alpha} X_{E L 1}(k) \cdot H(k)+\sqrt{1+\alpha} N(k)}{\hat{H}(k)}
\end{aligned}
$$

where $\hat{H}(k)$ expresses the estimated channel frequency response of the $k$-th sub-carrier. The bit data of the mobile reception can be obtained by decoding the $C L$ symbols, which then is reconstructed by BICM to generate $\widetilde{X}_{C L}(k)$. The demodulation of the EL1 symbols for fixed service can be expressed as follows:

$$
\begin{aligned}
\hat{X}_{E L 1}(k) & =\frac{Y_{L D M}(k) \sqrt{1+\alpha}}{\hat{H}(k) \sqrt{\alpha}}-\frac{1}{\sqrt{\alpha}} \widetilde{X}_{C L}(k) \\
& =X_{E L 1}(k) \cdot \frac{H(k)}{\hat{H}(k)}+\frac{\Delta_{C L}(k)+\frac{\sqrt{1+\alpha}}{H(k)} N(k)}{\sqrt{\alpha}} \\
\Delta_{C L} & (k)=X_{C L}(k) \frac{H(k)}{\hat{H}(k)}-\widetilde{X}_{C L}(k)
\end{aligned}
$$

where $\Delta_{C L}(k)$ denotes the remaining $C L$ signals after interference cancellation. Similarly, the $E L 2$ symbols, represented by $\hat{X}_{E L 2}(l)$, can be demodulated by performing the single-tap frequency-domain equalization. The complete $E L$ symbols for fixed service are obtained by combining EL1 and EL2 in the order of puncturing. Eventually, the bit data for fixed reception can be acquired by BICM decoding the $E L$ symbols.

The transmitter block diagram of LDM-Ex-FDM is shown in Figure 2. In Figures 2 and 3, in addition to the modules defined in the traditional $L D M$ scheme, the additional modules with full-color shadows are added to handle fixed service. The operating mechanism of the puncturing module in the additional module is detailed below. In this paper, the BICM encoder are used to generate quadrature phase-shift keying (QPSK) and non-uniform quadrature amplitude modulation (NU-QAM). The mobile service is carried by the CL of the $L D M$ while the fixed service is carried by the EL. Figure 4 shows the representation of CL, EL1, EL2 in the multiplexer (Mux) output, followed by subcarriers mapping, pilot insertion, inverse fast Fourier transform (IFFT) used in the frequency domain, and CP padding. Finally, the composite LDM-Ex-FDM signal is formed and transmitted over the air interface.

The receiver block diagram of LDM-Ex-FDM is shown in Figure 4. Firstly, after the signal is synchronized in time and frequency, $\mathrm{CP}$ removal and FFT, the LDM symbols and non- $L D M$ symbols can be obtained with frequency domain de-multiplexing, which are then stored respectively as "Buffering1" and "Buffering2". After that, the LDM symbols can be used to directly decode the bit data of the $C L$ which then is re-constructed through $\mathrm{BICM}$ to generate the $C L$ symbols. Moreover, $E L 1$ symbols for fixed service are obtained 
by subtracting the $C L$ symbols from "Buffering1" $L D M$ symbols. The complete $E L$ signal, which is the data symbols of the fixed service before puncturing, can be formed by combining EL1 symbols and EL2, the "Buffering2" non-LDM symbols. Finally, the bit data of fixed service is acquired by decoding the data symbols of fixed service.

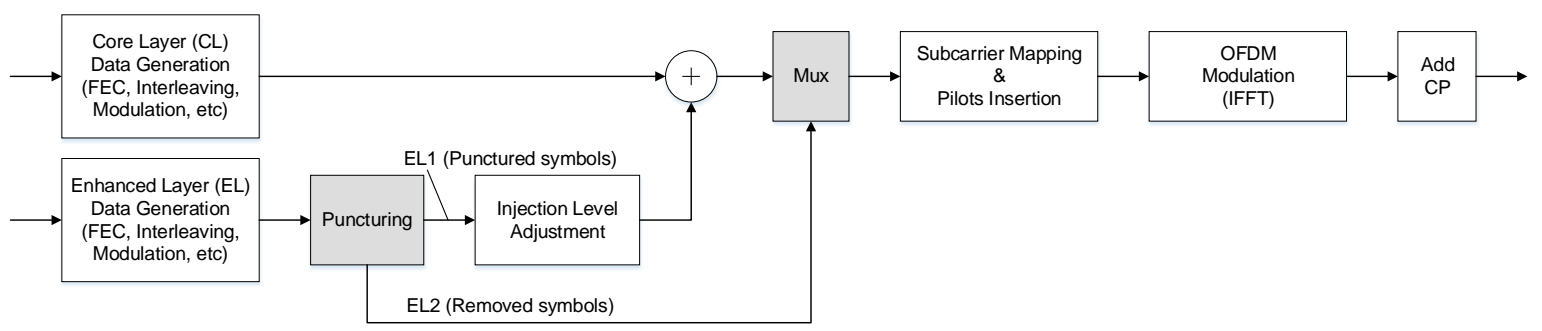

Figure 2. The transmitter structure of the layered division multiplexing extension frequency-division multiplexing (LDMEx-FDM) scheme.

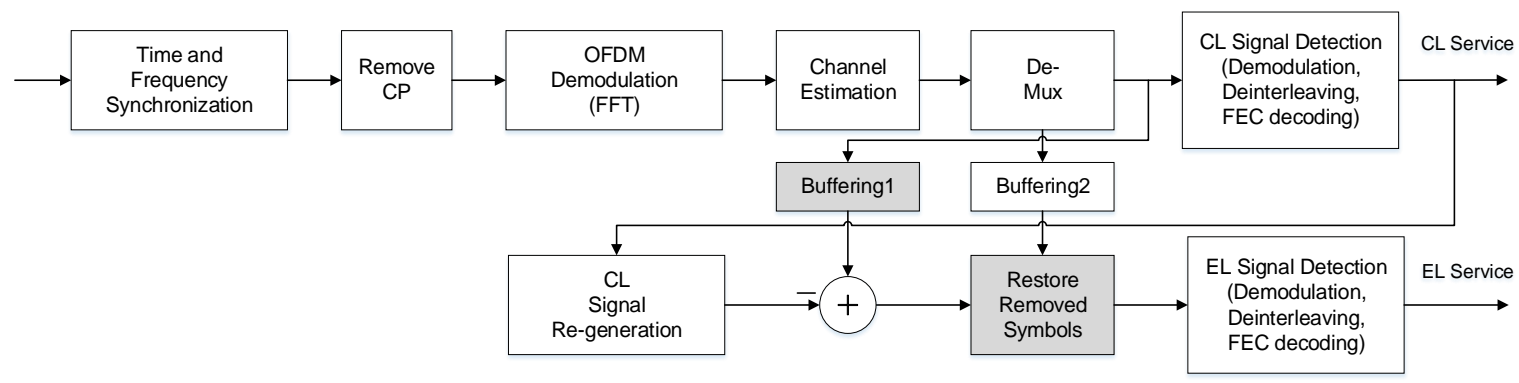

Figure 3. The receiver structure with the successive interference cancellation (SIC) detector of the LDM-Ex-FDM scheme.

\begin{tabular}{|c|c|}
\hline$X_{C L}$ & \\
\hline$X_{E L 1}$ & $X_{E L 2}$ \\
\hline & \\
\hline
\end{tabular}

Figure 4. The representation of core layer $(C L)$, enhanced layer $1(E L 1), E L 2$ in the multiplexer (Mux) output.

\subsection{Puncturing Strategy}

At the transmitter end, the puncturing strategy based on BICM symbols of the LDM-Ex-FDM scheme is divided into two types, namely EISP and NEISP, as shown in Figures 5 and 6, respectively. The EL symbols used for fixed reception are modulated by $\mathrm{BICM}$, and the modulated symbol puncturing accounts for $1 / 12$ of the total. Symbol puncturing at equal intervals can be used for multi-service transmission in typical scenarios. Symbol puncturing at non-equal intervals can be used for multi-service transmission in scenarios where the $L D M$ symbol constellation overlaps due to situations such as low power injection levels. The overlapping phenomenon of the constellation will reduce the service performance of mobile reception, resulting in the inability to completely eliminate the failure of the $C L$ signal, thereby further reducing the service performance of the fixed reception. However, the overlap phenomenon of constellations usually exists when the power injection level of the $L D M$ is small and/or the modulation order of $E L$ symbols is high.

The constellation comparison of the LDM symbols with and without NEISP in the LDM-Ex-FDM scheme is shown in Figure 7. When the power injection level of the LDM is $0 \mathrm{~dB}$, and the symbols of the $C L$ and $E L$ are respectively modulated by QPSK and NU64QAM, the constellations of the LDM symbols overlap. Take the constellation marked 
by the blue upper triangle as an example. It is not known in advance which symbol of $C L$ will be superimposed on the $E L$ symbol, that is, it is uncertain in which quadrant it will appear. As a result, symbols of non-overlapping constellations will also be punctured when puncturing according to high-power symbols. Specifically, the selection of removed symbols in the NEISP algorithm is as described in Algorithm 1.

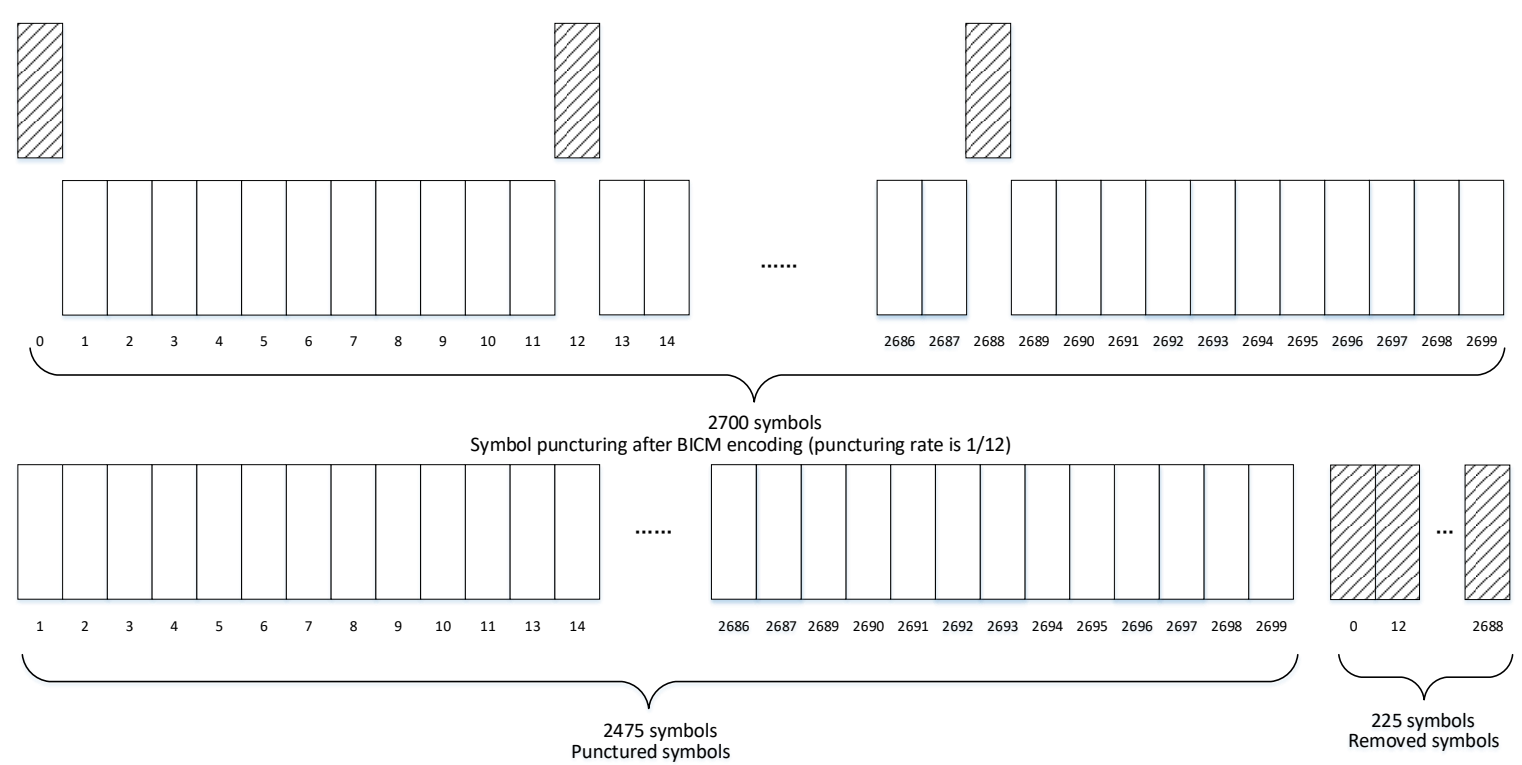

Figure 5. The schematic diagram of the equal interval symbol puncturing (EISP) after bit-interleaved coded modulation (BICM) encoding in the LDM-Ex-FDM scheme.

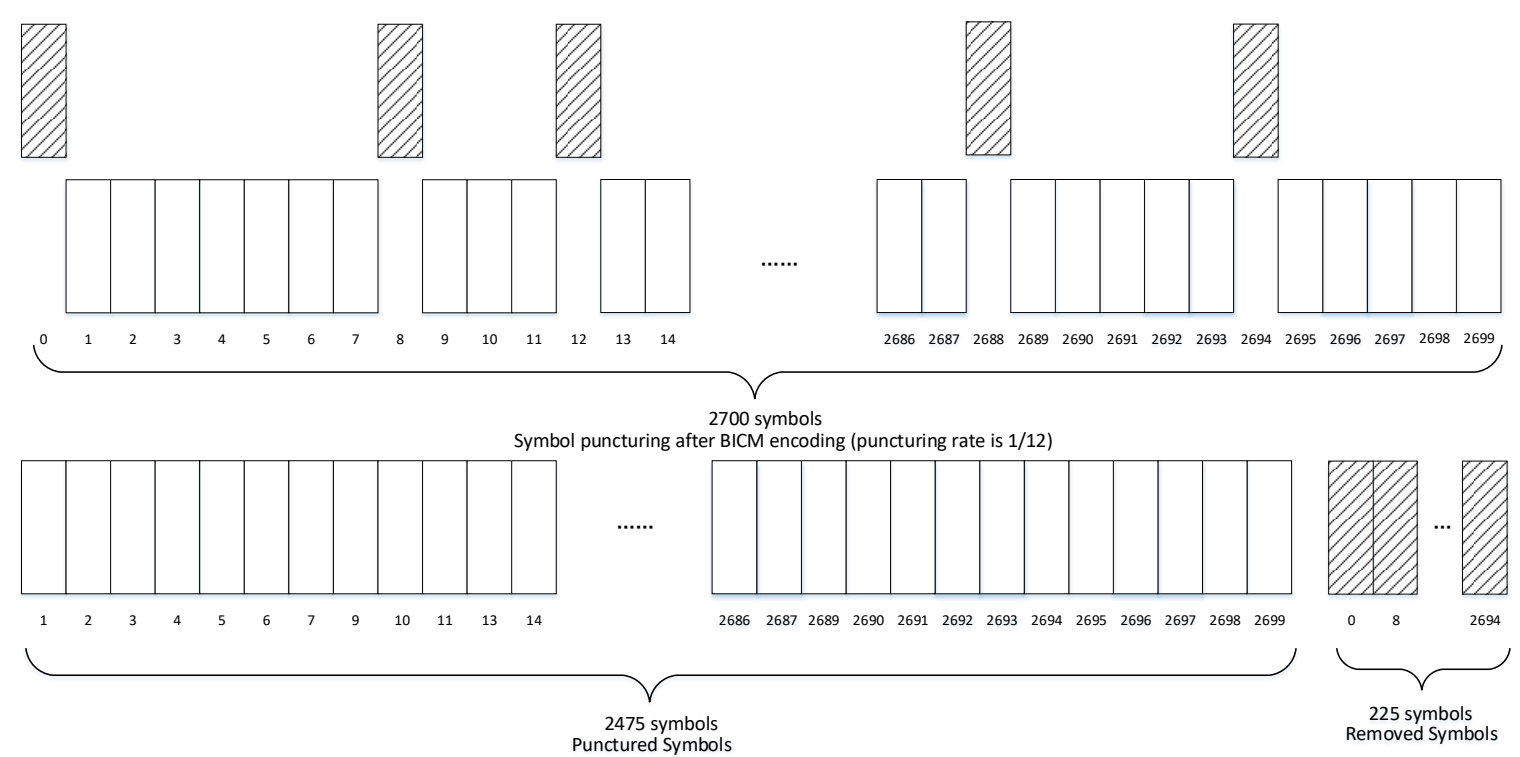

Figure 6. The schematic diagram of the non-equal interval symbol puncturing (NEISP) after BICM encoding in the LDM-Ex-FDM scheme. 


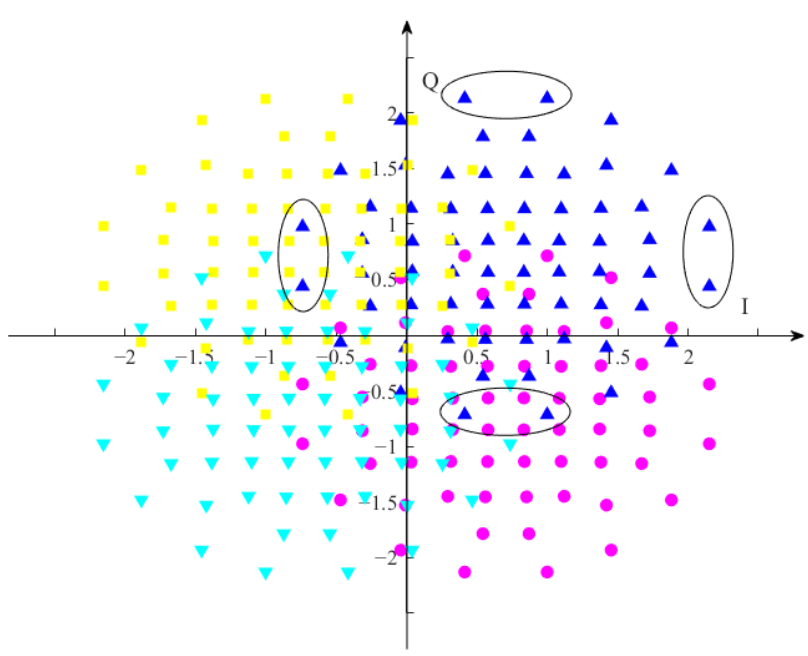

(a)

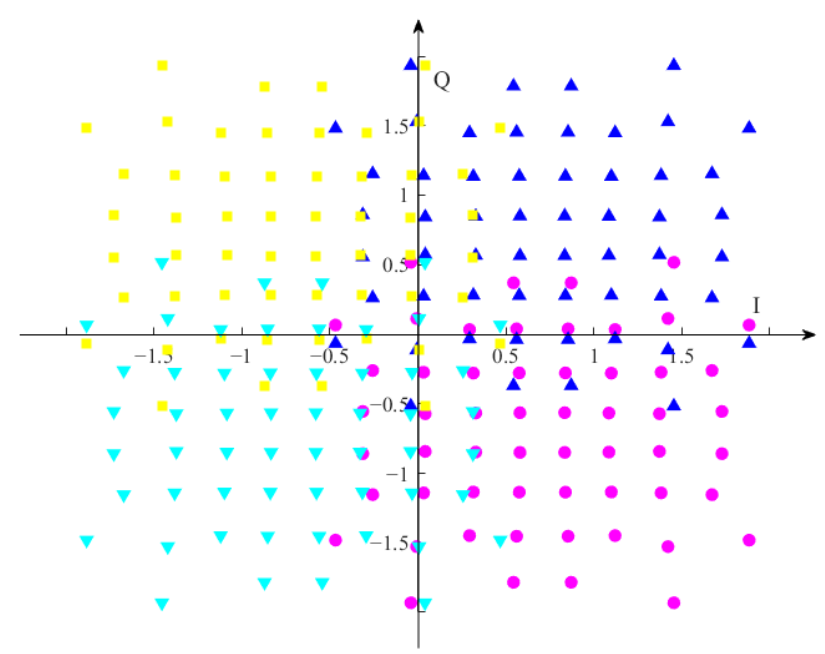

(b)

Figure 7. Comparison of the constellation of the LDM symbols with and without NEISP in the LDM-Ex-FDM scheme, where the injection level was set to $\Delta=0 \mathrm{~dB}$, the $C L$ constellation was set to quadrature phase-shift keying (QPSK), and the EL constellation was set to NU-64QAM (non-uniform quadrature amplitude modulation): (a) LDM symbol constellation before puncturing; (b) LDM symbol constellation after puncturing.

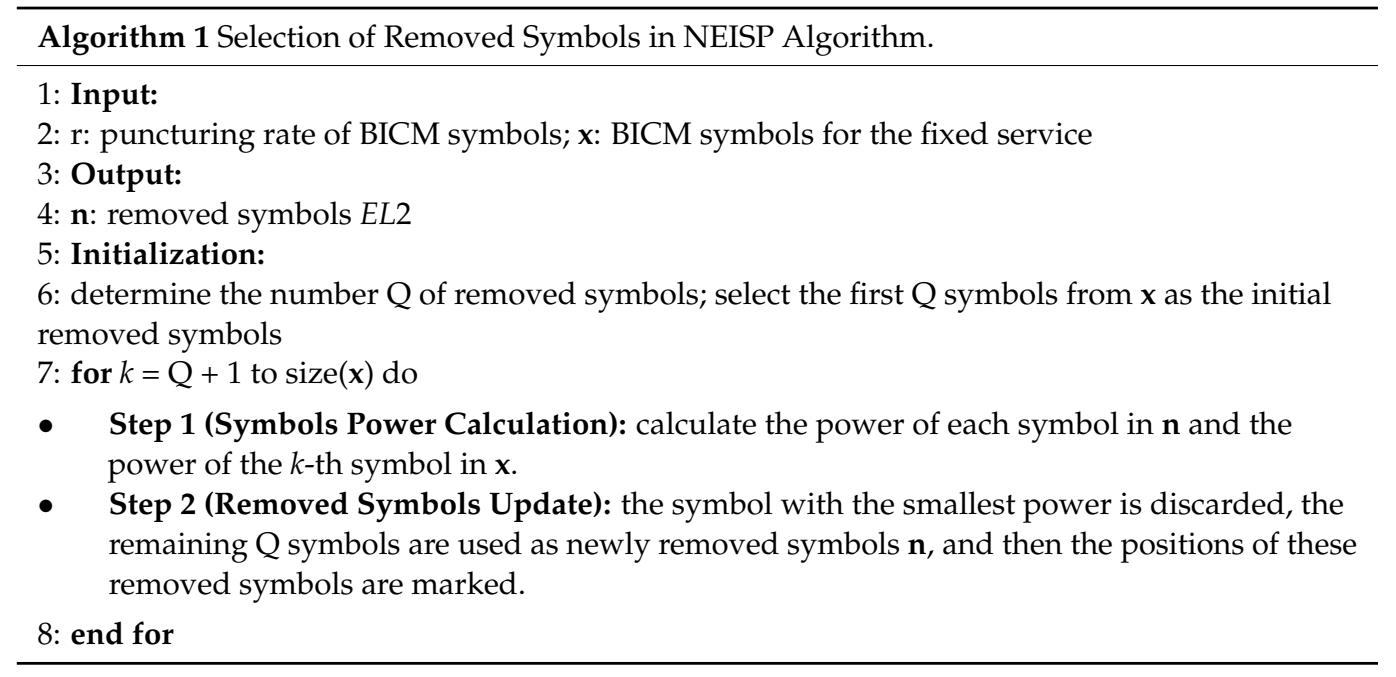

\subsection{Direct-Interference-Cancellation Scheme}

For fixed receivers, usually only the EL signal in the two-layer LDM is demodulated. To solve this problem, this paper proposes a novel DIC scheme. Note that in the DIC scheme, QPSK modulation is adopted for the CL of the LDM signal. Actually, to ensure the robustness of $C L$, the $C L$ of $L D M$ is usually equipped with low code rate FEC coding and a low-order modulation scheme. By adopting QPSK modulation and appropriate code rate, the 720p mobile service of $C L$ transmission can be sufficiently fulfilled. If the $C L$ adopts non-uniform 16QAM or other higher-order modulation, further optimization is needed for this scheme.

The core idea of DIC is to treat the LDM symbols as the EL symbols with constellation offset. The procedure of the proposed DIC scheme can be divided into the following steps: (1) Determine the distance of the constellation offset of the LDM symbol after channel equalization. If the equalized symbol constellation is within the first quadrant, the constellation offset distance will be determined as $\sqrt{2}+j \sqrt{2}$. Similarly, if the equalized symbol constellation is within the second, third, and fourth quadrants, the constellation offset distance will be determined as $-\sqrt{2}+j \sqrt{2},-\sqrt{2}-j \sqrt{2}$, and $\sqrt{2}-j \sqrt{2}$, respectively. (2) The second step is to subtract the corresponding constellation offset from the equalized 
$L D M$ symbol to obtain the repaired $E L$ symbol. (3) The third step is to pass the repaired $E L$ symbol through the BICM decoder to decode the EL bit data out.

Compared to the traditional SIC scheme, the demodulation of $C L$ symbols and the re-construction of $X_{C L}(k)$ can be omitted in the DIC scheme during the process of demodulating $E L$ symbols, thus the computational complexity and the processing delay can be significantly reduced at the receiver. In fact, the constellation offset $X_{C L}{ }^{\prime}(k)$ here can be regarded as the reconstructed signal $\widetilde{X}_{C L}(k)$ in the above SIC scheme. Furthermore, the process of repairing the constellation offset of the $E L$ signal in DIC is equivalent to eliminating the interference of $C L$ to the $E L$ signal in SIC.

According to (5), at the receiver, the demodulated EL symbol can be rewritten as:

$$
\begin{array}{r}
X_{E L}{ }^{\prime}(k)=\frac{\gamma_{L D M}(k) \sqrt{1+\alpha}}{\hat{H}(k) \sqrt{\alpha}}-\frac{1}{\sqrt{\alpha}} X_{C L}{ }^{\prime}(k) \\
=X_{E L}(k) \cdot \frac{H(k)}{\hat{H}(k)}+\frac{\Delta_{C L^{\prime}}(k)+\frac{\sqrt{1+\alpha}}{H(k)} N(k)}{\sqrt{\alpha}} \\
\Delta_{C L}{ }^{\prime}(k)=X_{C L}(k) \frac{H(k)}{\hat{H}(k)}-X_{C L}{ }^{\prime}(k)
\end{array}
$$

where $\Delta_{C L}{ }^{\prime}(k)$ indicates the error of repairing the constellation offset of the $E L$ signal. The bit data transmitted by $E L$ can be obtained through BICM decoding of the demodulated symbols of $E L$.

The receiver structure of the DIC scheme for demodulating $E L$ signal is shown in Figure 8, while the structure of the receiver for demodulating the $C L$ signal can be referred to Figure 1. The processing procedure of the receiver is as follows: after the signal is synchronized with time and frequency, CP removal, FFT and channel equalization, the demodulated $E L$ signal with constellation offset will be repaired using the proposed DIC scheme. Then, the repaired $E L$ symbol will be decoded by a BICM decoder to obtain the $E L$ bit data.

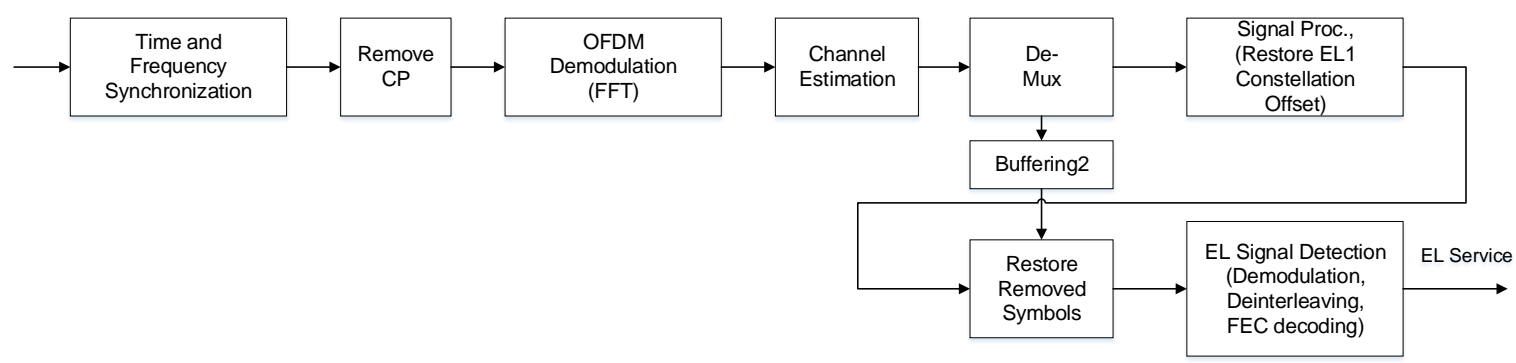

Figure 8. The receiver structure with the direct interference cancellation (DIC) detector of the LDM-Ex-FDM scheme.

\subsection{Bit-Interleaved Coded Modulation (BICM) Capacity Analysis}

BICM can be used to evaluate system performance in a binary communication system, which is an effective solution approaching Shannon's limit with affordable complexity. BICM capacity can be calculated by [19]:

$$
\begin{gathered}
C_{B, \chi}=\sum_{i=1}^{m} I\left(b_{i} ; Y\right) \\
I\left(b_{i} ; Y\right)=1-E_{b, y}\left[\log _{2} \frac{\sum_{x \in \chi} p(y \mid x)}{\sum_{x \in \chi_{b}^{i}} p(y \mid x)}\right]
\end{gathered}
$$

where $m$ is the order of the constellation, $I(\cdot \mid \cdot)$ denote average mutual information (AMI) function, $b_{i}$ denotes the $i$-th bit carried by a constellation symbol, $Y$ is the channel output. $\chi$ represents the alphabet of size $M=2^{m}, \chi_{b}^{i}$ represents a subset of all signals $x \in \chi$, and its tag has a value $b \in\{0,1\}$ at the $i$-th bit. $p(y \mid x)$ is the transition probability density function 
of the transmitted signal $x$ and the received signal $y . E_{b, y}[\cdot]$ is the expectation respect to the distribution $E$ and $y$.

$L D M$ enables the simultaneous delivery of mobile services and fixed services in the same time-frequency resources, the $C L$ signal power is regarded as the transmission power of the desired signal, and the EL signal power is regarded as the noise power when calculating the $C L$ channel capacity. In the ATSC 3.0 system with LDM-Ex-FDM scheme, the data symbol is divided into the $L D M$ symbol and the non- $L D M$ symbol part, and the AMI of the mobile service of the LDM symbol part can be calculated by [20]:

$I^{\prime}\left(b_{i} ; Y\right)=1-\frac{1}{2\left|\chi_{C} \| \chi_{E}\right|} \sum_{\chi_{C}} \sum_{\chi_{E}} \sum_{b} E_{N}\left[\log _{2} \frac{\sum_{x^{\prime} \in \chi_{C}} \exp \left(-\left(\left\|\rho \sqrt{\frac{1}{1+\alpha}} x_{C}+\rho \sqrt{\frac{\alpha}{1+\alpha}} x_{E}+N-\rho \sqrt{\frac{1}{1+\alpha}} x_{C}^{\prime}\right\|^{2}\right) / 2 \sigma^{2}\right)}{\sum_{x^{\prime \prime} \in \chi_{C, i}^{b}} \exp \left(-\left(\left\|\rho \sqrt{\frac{1}{1+\alpha}} x_{C}+\rho \sqrt{\frac{\alpha}{1+\alpha}} x_{E}+N-\rho \sqrt{\frac{1}{1+\alpha}} x^{\prime \prime}\right\|^{2}\right) / 2 \sigma^{2}\right)}\right]$

where $\rho \equiv 1$ under the additive white Gaussian noise (AWGN) channel, $\rho \sim C N(0,1)$ under the Rayleigh channel, and $N \sim C N\left(0, \sigma^{2}\right)$.

The AMIs of the fixed service of the $L D M$ symbol part and the non- $L D M$ symbol part can be calculated by Equation (10) because the $C L$ signal of the $L D M$ can be eliminated perfectly.

According to (9)-(11), the capacities of the mobile service and the fixed service in ATSC 3.0 system with and without LDM-Ex-FDM scheme over the AWGN and Rayleigh channels respectively are calculated and presented in Figures 9 and 10, respectively. The capacity improvement of the fixed service is expected to be $0.410 / 0.427 \mathrm{bit} / \mathrm{symbol}$ under the AWGN and Rayleigh channels respectively with the puncturing rate of the LDM-Ex-FDM scheme is $1 / 12$. Although the capacity of the mobile service has declined slightly, the capacity improvement of the total service (i.e., mobile service and fixed service) is expected to be $0.244 / 0.285 \mathrm{bit} / \mathrm{symbol}$ under the AWGN and Rayleigh channels, respectively. The reason is that the symbols removed by puncturing the fixed service symbols independently occupy a certain number of subcarriers for transmission, which reduces the transmission bandwidth of the mobile service. Obviously, the channel capacity of the fixed service and the overall service can be further improved by increasing the puncturing rate, but there needs to be a compromise between the performance improvement of the fixed service and the transmission rate of the mobile service. It is worth noting that the spectrum efficiency of the mobile service in the LDM-Ex-FDM scheme remains identical compared to the traditional LDM scheme, while the spectrum efficiency of the fixed service is higher.

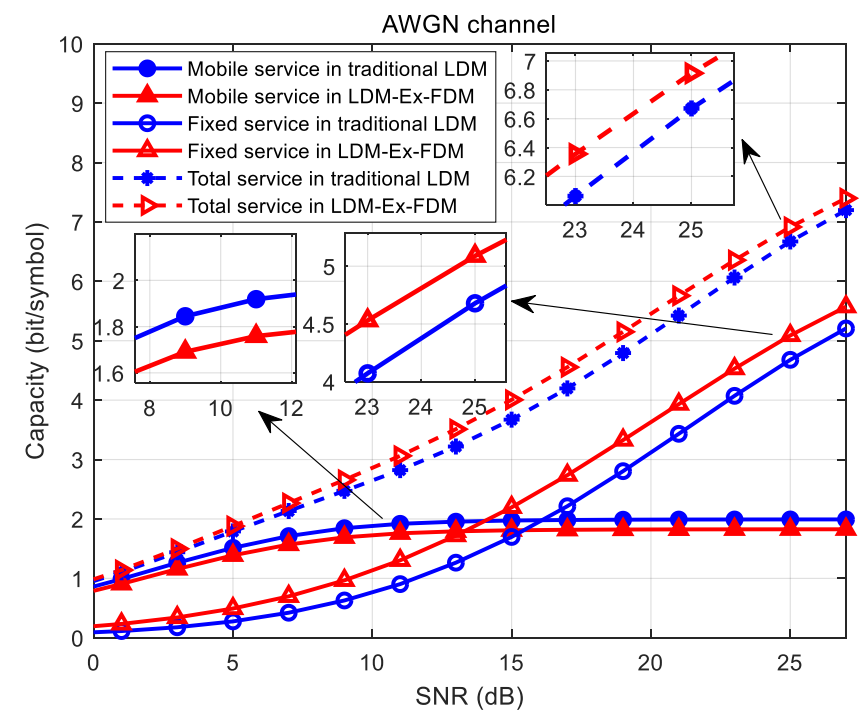

Figure 9. Capacities of mobile service and fixed service in Advanced Television System Committee (ATSC) 3.0 system with and without LDM-Ex-FDM scheme over the additive white Gaussian noise (AWGN) channel, where the parameter of ATSC 3.0 system was set to $\Delta=10 \mathrm{~dB}$, the $C L$ constellation was set to QPSK, the EL constellation was set to NU-64QAM, and the puncturing rate of the LDM-Ex-FDM scheme was set to $1 / 12$. 


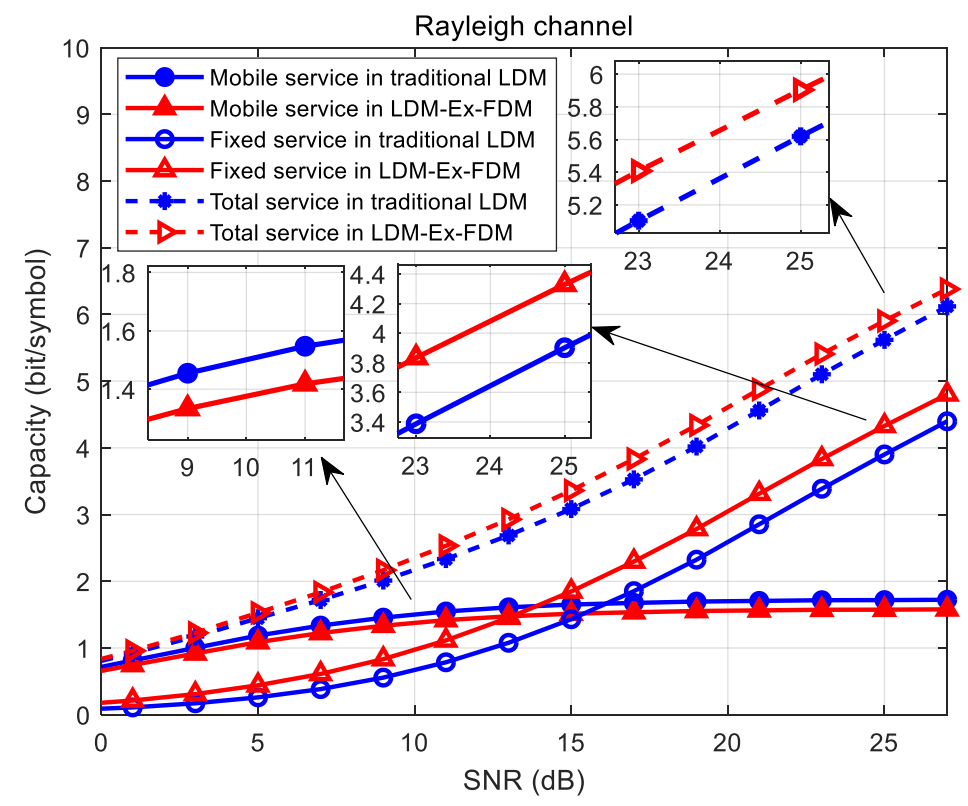

Figure 10. Capacities of mobile service and fixed service in ATSC 3.0 system with and without LDM-Ex-FDM scheme over the Rayleigh channel, where the parameter of ATSC 3.0 system was set to $\Delta=10 \mathrm{~dB}$, the $C L$ constellation was set to QPSK, the EL constellation was set to NU-64QAM, and the puncturing rate of the LDM-Ex-FDM scheme was set to $1 / 12$.

\section{Simulation Results and Analysis}

\subsection{Simulation Setup and Parameters}

In this section, the BER performance of the traditional LDM scheme was evaluated by physical layer simulations. The transceiver chain complied with the ATSC $3.0 \mathrm{spec}-$ ification [21]. The traditional SIC detector with a minimum mean square error (MMSE) equalizer was used to evaluate performance. Two symbol puncturing algorithms and the DIC detector described in Section 3 were implemented. The scattered pilot (SP) scheme shown in Figure 11 was utilized in the simulation. The proposed LDM-Ex-FDM scheme was compared with the traditional ATSC 3.0 scheme in terms of BER.

The channel models used in the simulator included the AWGN channel and multipath fading channel for both mobile and fixed reception scenarios. The multipath fading channel was a fast-fading model composed of six taps shown in Table 1 . Both the ideal and least square (LS) channel estimation methods were adopted. Moreover, the ideal time and frequency synchronization was assumed in the simulation for the sake of simplicity.

The basic transmission parameters for simulations are shown in Table 2. To facilitate the development of the practical system with low complexity and low memory usage, the LDPC code length was 16,200 and the FFT size was $16 \mathrm{~K}$ for both the $C L$ and the $E L$ signals. In addition, in terms of modulation and code rate, the LDPC code rate used by $C L$ is $5 / 15$ and the constellation was QPSK because $C L$ is typically designed to deliver robust services to mobile receivers at a low SNR, while the LDPC code rate for the $E L$ was $11 / 15$ and the constellations were NU-16QAM and NU-64QAM instead due to the fact that $E L$ is typically designed to provide high data rate services to fixed receivers at a high SNR. The puncturing rate was chosen from $1 / 12$ to $1 / 6$, while the injection level varies from 10 to $15 \mathrm{~dB}$. The symbol-level SNR was used in the following part. The simulations were performed on the MATLAB 2017b platform with Intel(R) Xeon(R) E5-2667 central processing unit (CPU) under $2.9 \mathrm{GHz}$ and the 64-bit Windows 7 operating system. All results were obtained on the basis of 10,000 frames of Monte Carlo simulation per SNR. 


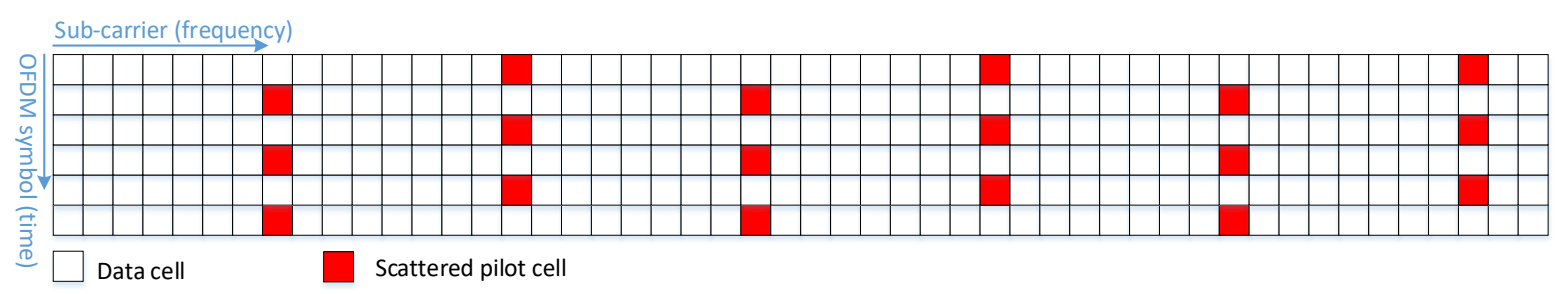

Figure 11. Scattered pilot (SP) orthogonal frequency division multiplexing (OFDM) subcarriers.

Table 1. Profile of the multipath channel model.

\begin{tabular}{ccc}
\hline Echo & Power $(\mathbf{d B})$ & Time Delay $(\mu \mathbf{s})$ \\
\hline 1 & -3 & 0 \\
2 & 0 & 0.2 \\
3 & -2 & 0.5 \\
4 & -6 & 1.6 \\
5 & -8 & 2.3 \\
6 & -10 & 5.0 \\
\hline
\end{tabular}

Table 2. Basic transmission parameters.

\begin{tabular}{cc}
\hline Parameter & Value \\
\hline Fast Fourier transform (FFT) size & $16 \mathrm{k}$ \\
Scattered pilot (SP) pattern & $\mathrm{SP} 8 \_2$ \\
Cyclic prefix $(\mathrm{CP})$ & $1 / 16$ \\
Core layer (CL) low-density parity-check & 16,200 \\
(LDPC) code length & $5 / 15$ \\
$C L$ LDPC code rate & 50 \\
$C L$ number of iterations & quadrature phase-shift keying (QPSK) \\
$C L$ constellation & 16,200 \\
Enhanced layer $(E L)$ LDPC code length & $11 / 15$ \\
$E L$ LDPC code rate & 50 \\
$E L$ number of iterations & non-uniform $(\mathrm{NU})-16$ quadrature amplitude \\
EL constellation & modulation $(\mathrm{QAM}), \mathrm{NU}-64 \mathrm{QAM}$ \\
Puncturing rate $(\gamma)$ & $1 / 6,1 / 12$ \\
Injection level $(\Delta)$ & $10 \mathrm{~dB}, 12 \mathrm{~dB}, 15 \mathrm{~dB}$ \\
\hline
\end{tabular}

\subsection{LDM-Ex-FDM Performance with Equal Interval Symbol Puncturing (EISP)}

This subsection simulates the multi-service transmission performance of the LDM-ExFDM scheme with EISP. The BER performance of the traditional scheme and the proposed scheme for receiving mobile services and fixed services in the AWGN channel is shown in Figures 12 and 13, respectively. In Figure 12, the SNR thresholds of mobile service of these two schemes are almost the same under the AWGN channel. It can be observed in Figure 13 that when the puncturing rate of BICM symbols for fixed service data is $1 / 12$, the BER performance of the newly proposed LDM-Ex-FDM transmission scheme outperforms that of the traditional LDM scheme by about $0.45 \mathrm{~dB} @ \mathrm{BER}=10^{-5}$ under the AWGN channel. This is due to the fact that the SNR of EL2 that occupies part of the subcarriers for FDM transmission is higher than that of $E L 1$ in $L D M$. Moreover, when the puncturing rate is set at $1 / 6$, the improvement of the BER performance of the proposed scheme is up to $0.9 \mathrm{~dB}$.

The BER performance for mobile services and fixed services in the multipath channel is shown in Figures 14 and 15, respectively. In Figure 14, the SNR thresholds of mobile service of these two schemes are almost the same in the multipath channel. In Figure 15, it is observed that the LDM-Ex-FDM scheme can achieve the BER performance improvement of up to $1.8 \mathrm{~dB} @ \mathrm{BER}=10^{-6}$ under the multipath channel when the puncturing rate is $1 / 12$, and improvement of about $2.5 \mathrm{~dB}$ when the puncturing rate is set at $1 / 6$. 
Another observation extracted from Figures 13 and 15 is that when the number of occupied subcarriers of $E L 2$ doubles, the SNR performance improvement is nearly doubled, i.e., from 0.45 to $0.9 \mathrm{~dB}$, under AWGN channel. In the multipath fading channel, however, when the number of occupied subcarriers of EL2 doubles, the BER performance gain is only increased by about $38 \%$, i.e., from 1.8 to $2.5 \mathrm{~dB}$. It can be inferred that the demodulation performance of fixed service can be significantly improved by selecting an appropriate number of occupied subcarriers.

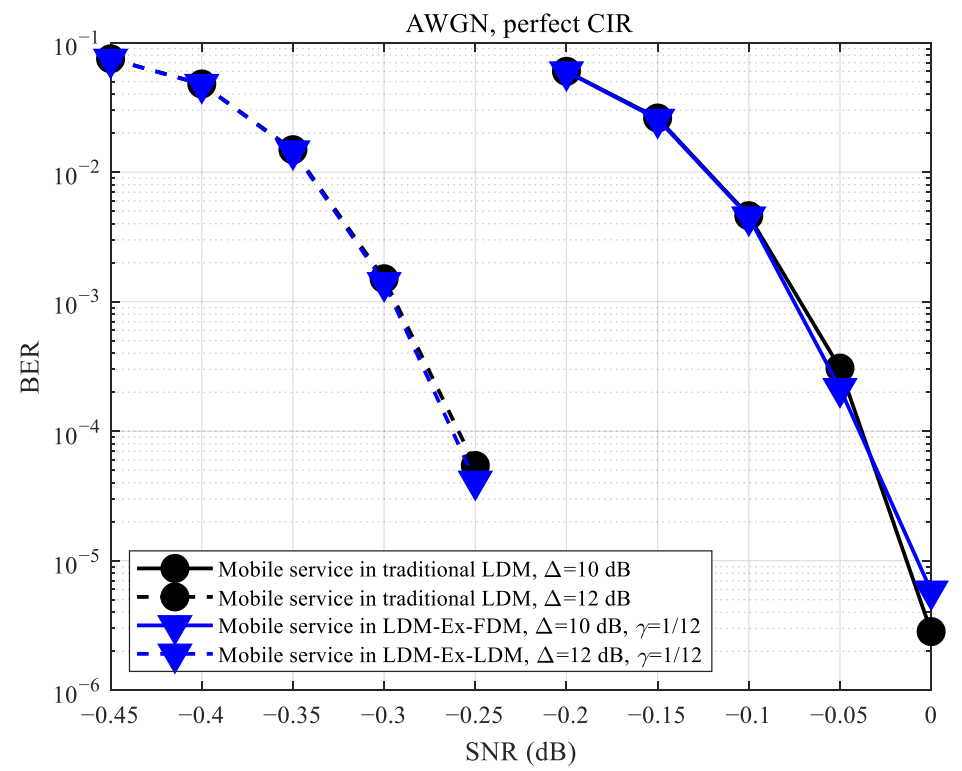

Figure 12. Bit error rate (BER) performance of mobile service in ATSC 3.0 system with and without LDM-Ex-FDM scheme with perfect channel impulse response (CIR) over the AWGN channel, where the parameters of the ATSC 3.0 system were set to $\Delta=10$ and $12 \mathrm{~dB}$, the $C L$ constellation was set to QPSK, the EL constellation was set to NU-64QAM, and the puncturing rate of the LDM-Ex-FDM scheme was set to $1 / 12$.

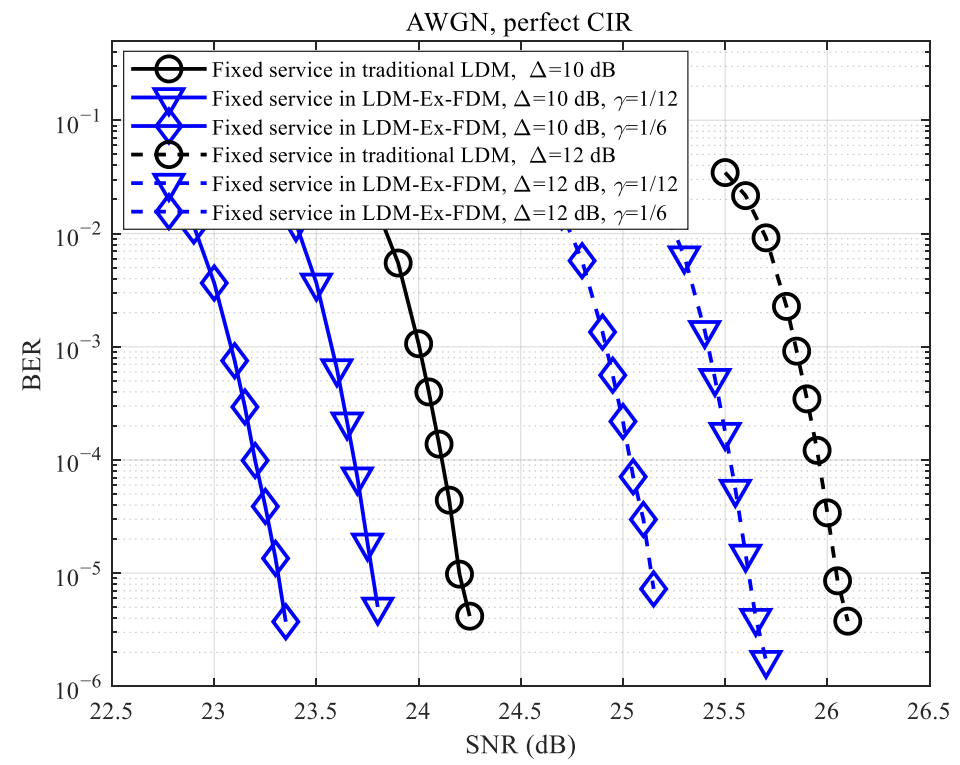

Figure 13. BER performance of fixed service in ATSC 3.0 system with and without LDM-Ex-FDM scheme with perfect channel impulse response (CIR) over the AWGN channel, where the parameters of the ATSC 3.0 system were set to $\Delta=10$ and $12 \mathrm{~dB}$, the CL constellation was set to QPSK, the EL constellation was set to NU-64QAM, and the puncturing rate of the LDM-Ex-FDM scheme were set to $1 / 6$ and $1 / 12$. 


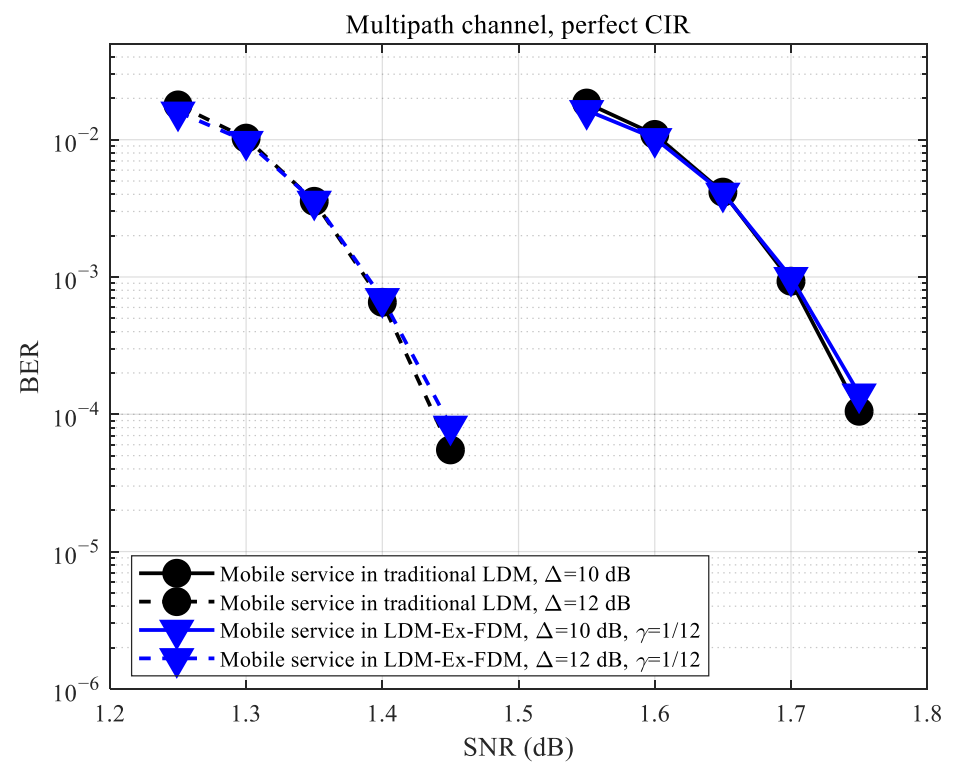

Figure 14. BER performance of mobile service in ATSC 3.0 system with and without LDM-ExFDM scheme with perfect channel impulse response (CIR) under the multipath channel, where the parameters of the ATSC 3.0 system were set to $\Delta=10$ and $12 \mathrm{~dB}$, the $C L$ constellation was set to QPSK, the EL constellation was set to NU-64QAM, and the puncturing rate of the LDM-Ex-FDM scheme was set to $1 / 12$.

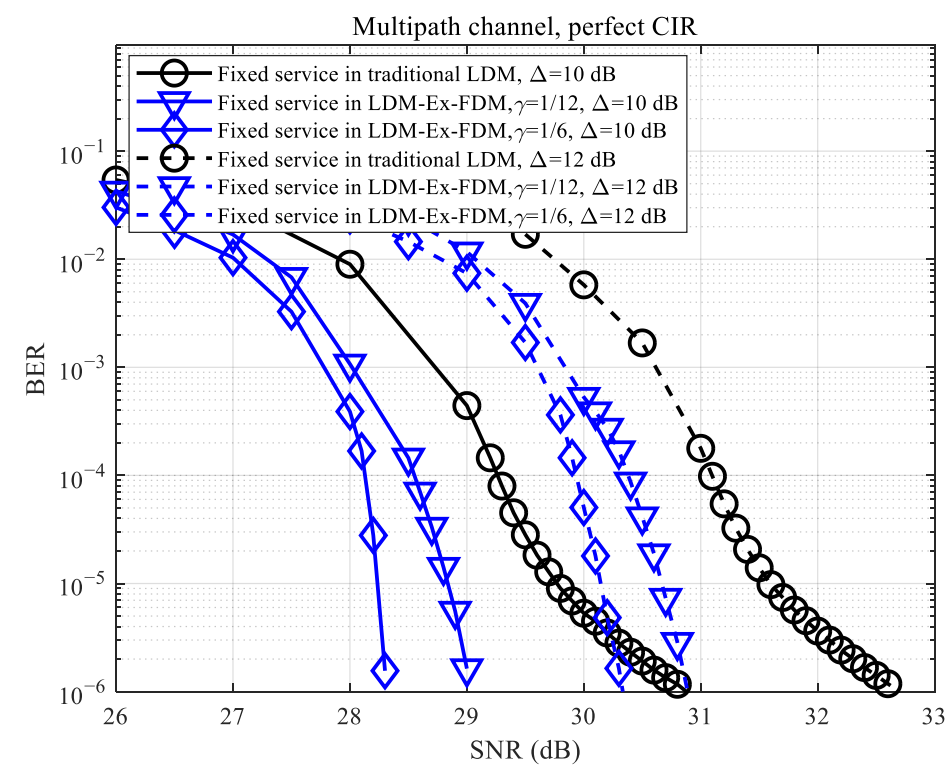

Figure 15. BER performance of fixed service in ATSC 3.0 system with and without LDM-Ex-FDM scheme with perfect channel impulse response (CIR) under the multipath channel, where the parameters of the ATSC 3.0 system were set to $\Delta=10$ and $12 \mathrm{~dB}$, the CL constellation was set to QPSK, the EL constellation was set to NU-64QAM, and the puncturing rate of the LDM-Ex-FDM scheme were set to $1 / 6$ and $1 / 12$.

As shown in Figures 16 and 17, the BER performance of each service in the LDM-ExFDM scheme and the traditional LDM scheme can be compared when the channel impulse response is estimated from the received data. Here, the pilot-assisted least square (LS) channel estimation algorithm is used. A conclusion similar to that shown in Figures 14 and 15 can be drawn from this. 


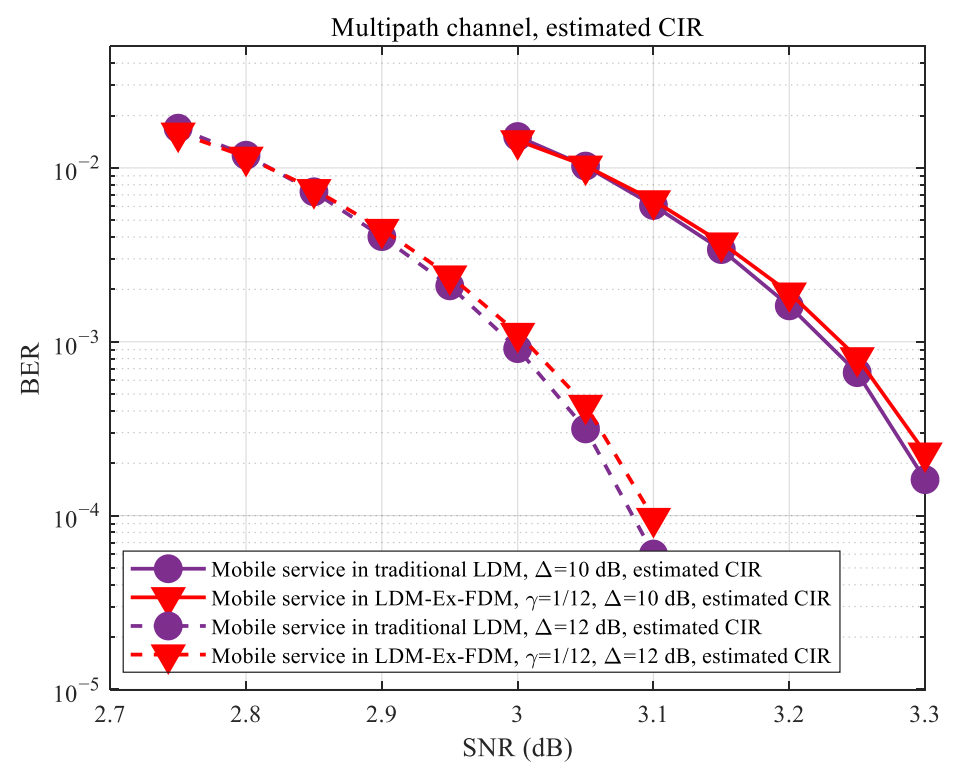

Figure 16. BER performance of mobile service in ATSC 3.0 system with and without LDM-Ex-Figure 3.0 system were set to $\Delta=10$ and $12 \mathrm{~dB}$, the CL constellation was set to QPSK, the EL constellation was set to NU-64QAM, and the puncturing rate of the LDM-Ex-FDM scheme was set to 1/12.

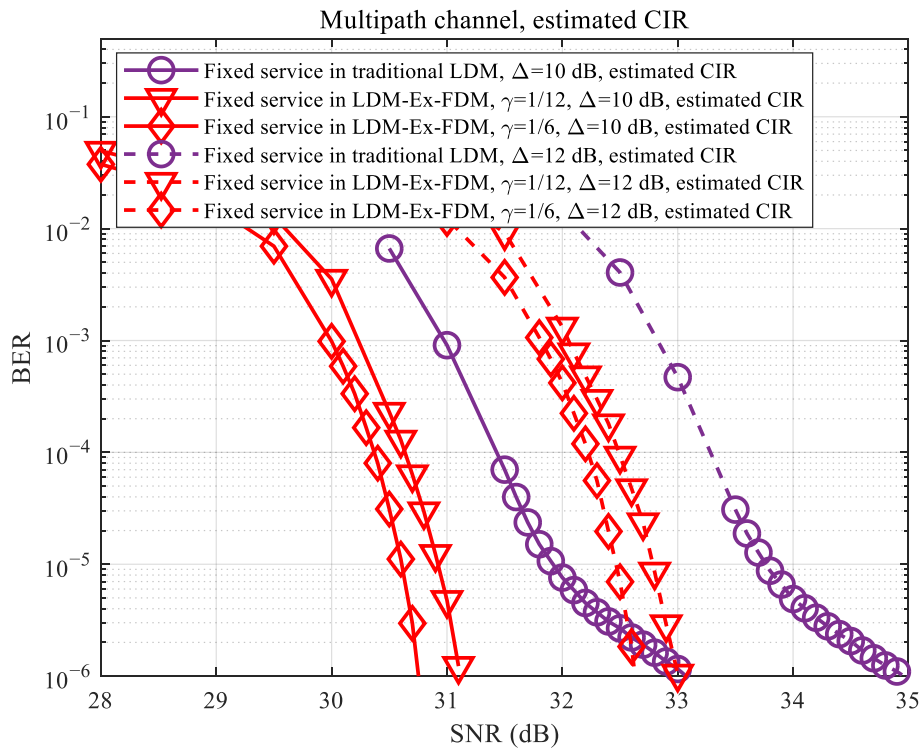

Figure 17. BER performance of fixed service in ATSC 3.0 system with and without LDM-Ex-FDM scheme with estimated channel impulse response (CIR) under the multipath channel, where the parameters of the ATSC 3.0 system were set to $\Delta=10$ and $12 \mathrm{~dB}$, the $C L$ constellation was set to QPSK, the EL constellation was set to NU-64QAM, and the puncturing rate of the LDM-Ex-FDM scheme were set to $1 / 6$ and $1 / 12$.

Compared to the traditional ATSC 3.0 scheme where all subcarriers are multiplexed in the power domain, the proposed LDM-Ex-FDM scheme keeps part of the subcarriers powermultiplexed, and part are frequency multiplexed. Although this will cause the transmission rate of the mobile service to drop slightly, it will not deteriorate the received SNR threshold of the $C L$ layer mobile service of $L D M$. On the other hand, adopting proper puncturing interval for BICM symbols of fixed service data can reduce the SNR demodulation threshold of fixed service data. This can be explained by the following example. Table 3 presents the comparison of the loss of the transmission rate for mobile service and the improvement of the required SNR for fixed services under the AWGN and multipath channels. In the case 
of QPSK 5/15, as shown in Table 3, in the AWGN channel, by sacrificing $0.27 \mathrm{Mbps}$ of the transmission rate for the $C L$ mobile service, the BER performance gain for the $E L$ service can be up to $0.45 \mathrm{~dB}$. Furthermore, it is possible to increase the BER performance for fixed services by $0.9 \mathrm{~dB}$ at maximum if the mobile service can accept a $0.54 \mathrm{Mbps}$ transmission rate loss. In this case, the mobile service can still reach a transmission rate of $2.67 \mathrm{Mbps}$, which is sufficient for the transmission of 720p mobile service in the typical scenario with LDM in the ATSC 3.0 system [16]. In the more practical multipath channel, the improvement of the received SNR threshold of $E L$ service can be up to $1.8 \mathrm{~dB}$ at the sacrifice of 0.27 Mbps of transmission rate loss for $C L$ mobile service. This indicates that the proposed LDM-Ex-FDM transmission scheme will have better performance in a practical system. Therefore, the proposed scheme can achieve a compromise between the demodulation performance of the fixed service and the data rate of the mobile service transmission.

Table 3. Bit rate for mobile service and the improvement of the BER performance for fixed service under the AWGN and multipath channels.

\begin{tabular}{ccc}
\hline Scheme & $\begin{array}{c}\text { Bit Rate for Mobile Service } \\
\text { (QPSK-5/15) }\end{array}$ & $\begin{array}{c}\text { Improvement of the BER Performance for Fixed Service } \\
\text { under AWGN/Multipath Channel }\end{array}$ \\
\hline Traditional LDM & $3.21 \mathrm{Mbps}$ & - \\
\hline $\begin{array}{c}\text { LDM-Ex-FDM } \\
\text { Puncturing Rate }=1 / 12\end{array}$ & $2.94 \mathrm{Mbps}$ & $0.45 \mathrm{~dB} / 1.8 \mathrm{~dB}$ \\
\hline $\begin{array}{c}\text { LDM-Ex-FDM } \\
\text { Puncturing Rate }=1 / 6\end{array}$ & $2.67 \mathrm{Mbps}$ & $0.90 \mathrm{~dB} / 2.5 \mathrm{~dB}$ \\
\hline
\end{tabular}

\subsection{LDM-Ex-FDM Performance with Non-Equal Interval Symbol Puncturing (NEISP)}

This subsection simulates the multi-service transmission performance of the LDMEx-FDM scheme with NEISP. The BER performance of the traditional LDM scheme and the proposed LDM-Ex-FDM scheme for receiving mobile service and fixed service in the multipath fading channel is shown in Figure 18.

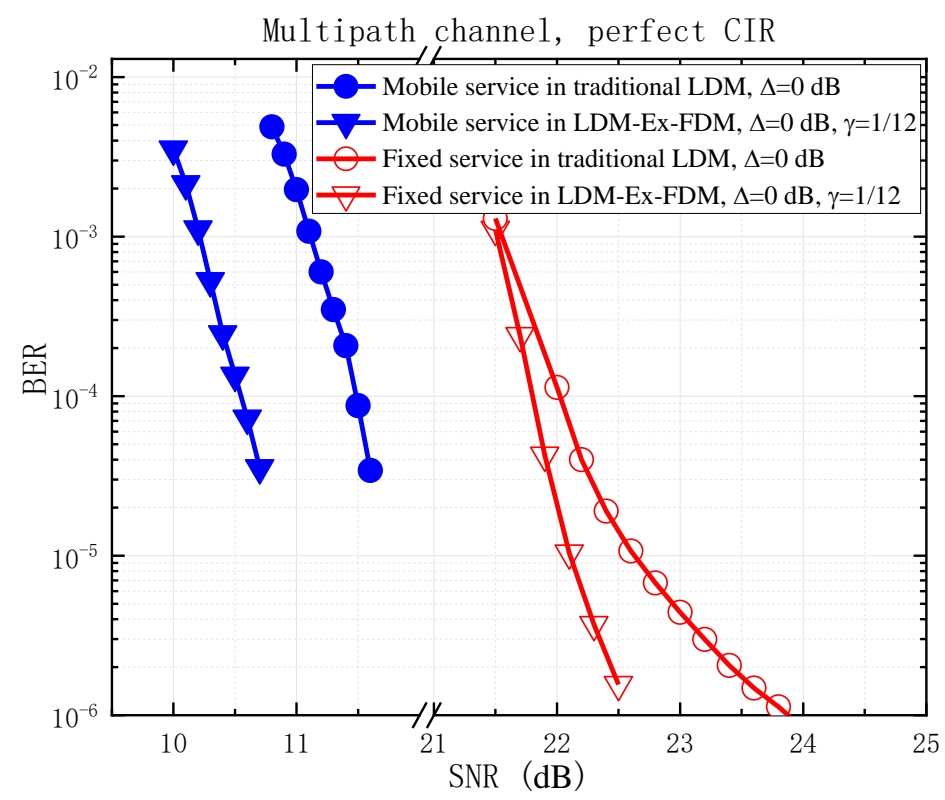

Figure 18. BER performance of mobile service and fixed service in ATSC 3.0 system with and without LDM-Ex-FDM scheme with perfect channel impulse response (CIR) under the multipath channel, where the parameters of the ATSC 3.0 system was set to $\Delta=0 \mathrm{~dB}$, the CL constellation was set to QPSK, the EL constellation was set to NU-64QAM, and the puncturing rate of the LDM-Ex-FDM scheme was set to $1 / 12$. 
The BER performance of the mobile service and the fixed service in the LDM-ExFDM multi-service transmission scheme proposed in this paper is better than that of the traditional $L D M$ scheme by approximately $[0.90,1.05] \mathrm{dB}$. This is due to the fact that the use of NEISP effectively reduces the occupancy rate of constellation overlap, thereby improving the performance of mobile services. On the other hand, the symbols removed by puncturing occupy part of the subcarriers transmission alone and therefore have a higher SNR at the receiving end, which can improve the decoding performance of the fixed service.

\subsection{DIC Performance for the Fixed Reception}

In this subsection, the BER performance comparisons of the LDM-Ex-FDM scheme with the proposed DIC scheme and the traditional SIC scheme are shown in Figures 19 and 20, respectively. Note that the proposed DIC could also work with traditional LDM used in ATSC 3.0. As shown in Figure 19, the BER performance of the proposed LDM-Ex-FDM scheme with NU-16QAM constellation mapping and a DIC detector under the multipath channel when different power injection levels of $L D M$ are allocated. It can be observed that when the power injection level is $10 \mathrm{~dB}$, there exists a gap of about $0.1 \mathrm{~dB} @ \mathrm{BER}=10^{-6}$ between the low-complexity DIC detector and the SIC scheme. Moreover, when the power injection level is higher than $10 \mathrm{~dB}$, the performance loss reduces to within $0.1 \mathrm{~dB}$. In Figure 20, when a higher-order modulation, NU-64QAM, is applied in the fixed service, the proposed DIC scheme is capable of achieving almost the same BER performance as that of the traditional SIC. It can be inferred from Figures 19 and 20 that the higher the power injection level and/or the modulation order adopted by the fixed service, the better performance that the DIC scheme can achieve.

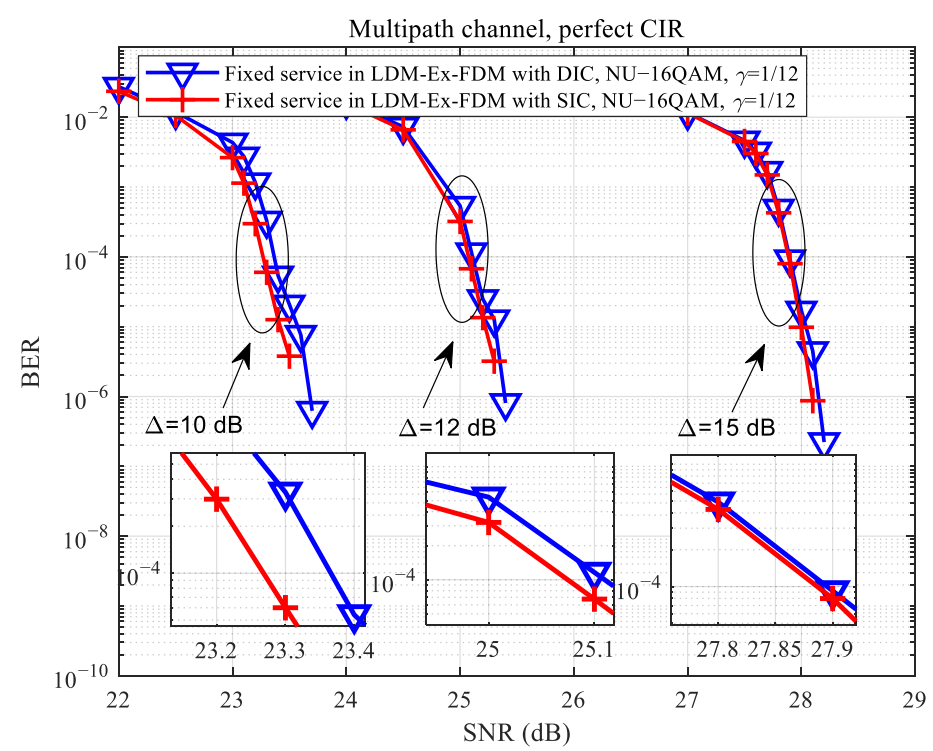

Figure 19. BER performance of fixed service in the LDM-Ex-FDM scheme with direct interference cancellation (DIC) and successive interference cancellation (SIC) with perfect channel impulse response (CIR) under the multipath channel, where the parameters of the ATSC 3.0 system were set to $\Delta=10 \mathrm{~dB}, 12 \mathrm{~dB}, 15 \mathrm{~dB}$, the $C L$ constellation was set to QPSK, the EL constellation was set to $\mathrm{NU}-16 \mathrm{QAM}$, and the puncturing rate was set to $1 / 12$. 


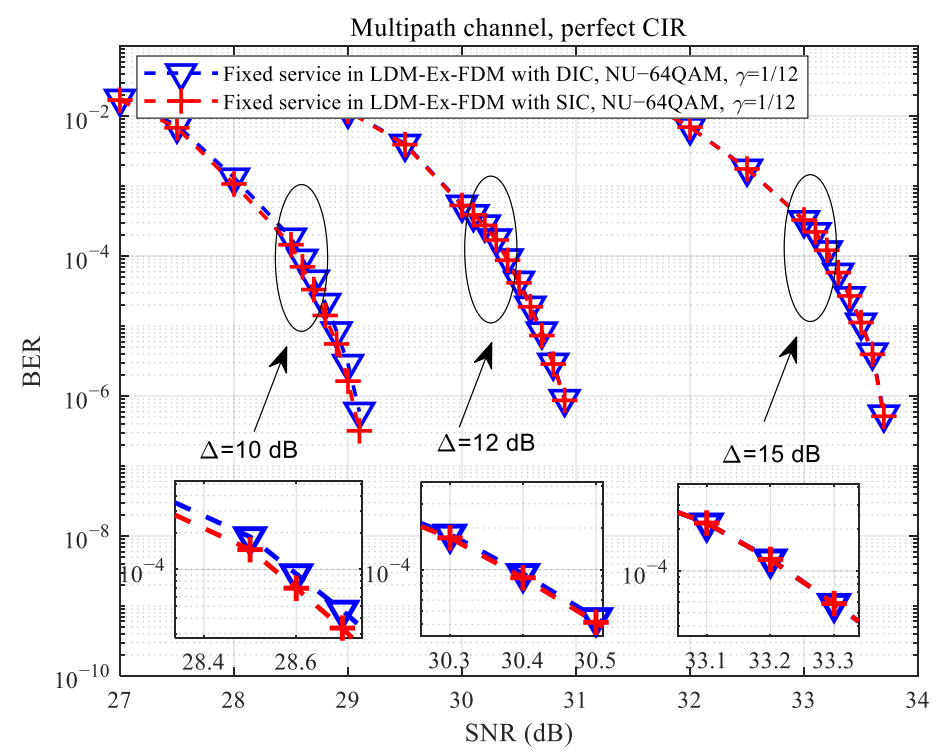

Figure 20. BER performance of fixed service in the LDM-Ex-FDM scheme with DIC and SIC with perfect channel impulse response (CIR) under the multipath channel, where the parameters of the ATSC 3.0 system were set to $\Delta=10 \mathrm{~dB}, 12 \mathrm{~dB}, 15 \mathrm{~dB}$, the $C L$ constellation was set to QPSK, the $E L$ constellation was set to NU-64QAM, and the puncturing rate was set to $1 / 12$.

The basic principle of DIC is that it regards the $C L$ symbols as the constellation offset of the $E L$ symbols of the $L D M$ symbols, and demodulates the $C L$ symbols by making a hard decision on the composite constellation of the $L D M$ symbols. Therefore, the composite constellation of $L D M$ symbols has nothing to do with the signal coding type in each layer of $L D M$, but is affected by the modulation type and the power injection level of the signal in each layer of $L D M$.

As shown by these results, compared to the SIC scheme, the proposed DIC scheme can achieve lower processing complexity and lower delay with almost no performance loss in the case of a relatively high power injection level.

\subsection{Further Discussions on the LDM-Ex-FDM Scheme}

Based on the above simulation results and the BICM capacity analysis in Section 3, it is shown that the LDM-Ex-FDM scheme has a higher EL capacity and the overall capacity than the traditional $L D M$ scheme. Compared to the traditional method, i.e., to increase the $E L$ power by simply changing the $L D M$ power injection level, the proposed solution is able to improve the decoding performance of the fixed service without decreasing the required SNR threshold for mobile services. This can be explained by the effective use of the puncturing strategies at the transmitter with which part of the EL services can be demodulated with less signal interference and a higher receiving SNR at the receiver side, so as to get more frequency diversity gain. It is noted that this capacity gain comes at the cost of increased complexity at the transmitter side. Therefore, a DIC detection method is developed to further reduce the overall computational complexity. The technical comparison between the proposed LDM-Ex-FDM scheme and several typical LDM schemes is illustrated in Table 4.

In fact, our solution is helpful for some practical application scenarios. Take the system coverage and networking into consideration, $L D M$ can achieve the same coverage requirements of different services by reasonably selecting the coding and modulation parameters and the injection level of both layers. However, in actual network deployment, when the antenna gain and installation height are not completely matched with the planned parameters and other non-ideal conditions, the effective coverage of the transmission service will be reduced, and in particular the coverage performance of the $E L$ will be worse as a result of a higher demodulation SNR threshold. In this case, multiple EL holes will 
be generated in the network coverage area. This phenomenon is more common in urban environments. The proposed LDM-Ex-FDM scheme is capable of improving the reception performance of the $E L$ services without degrading the reception SNR threshold of the $C L$ services, and further increase the overall system coverage performance. In a sense, the $E L$ coverage hole can be filled effectively.

Table 4. The comparison among several $L D M$ schemes.

\begin{tabular}{ccccc}
\hline Scheme & Transmitter & Receiver & $\begin{array}{c}\text { Achieved } \\
\text { Capacity }\end{array}$ & Complexity \\
\hline $\begin{array}{c}\text { layered division } \\
\text { multiplexing }(L D M)\end{array}$ & LDM & $\begin{array}{c}\text { successive } \\
\text { interference } \\
\text { cancellation (SIC) }\end{array}$ & low & low \\
\hline $\begin{array}{c}\text { time-layered } \\
\text { division } \\
\text { multiplexing } \\
\text { (TLDM) }\end{array}$ & $\begin{array}{c}L D M \text {, time-division } \\
\text { multiplexing (TDM) }\end{array}$ & SIC & medium & medium \\
\hline $\begin{array}{c}\text { layered division } \\
\text { multiplexing } \\
\text { extension } \\
\text { frequency-division } \\
\text { multiplexing } \\
\text { (LDM-Ex-FDM) }\end{array}$ & $\begin{array}{c}\text { multiplexing (FDM), } \\
\text { equal interval } \\
\text { symbol puncturing } \\
\text { (EISP)/NEISP }\end{array}$ & $\begin{array}{c}\text { SIC/ direct } \\
\text { interference } \\
\text { cancellation (DIC) }\end{array}$ & high & medium \\
\hline
\end{tabular}

\section{Conclusions}

In this paper, a multi-service transmission scheme called LDM-Ex-FDM for the ATSC 3.0 system is proposed. The proposed scheme can support simultaneous transmission of multiple services with different received SNR thresholds.

First, the system's BICM capacity calculation shows that the LDM-Ex-FDM scheme can effectively increase the fixed service capacity and the overall service capacity while appropriately reducing the capacity of the mobile service. It should be noted that the reduction in the capacity of the mobile service is due to the reduction in the bandwidth used, thus the spectrum efficiency of the mobile service has not changed compared with the traditional LDM scheme. Second, the computer simulation results show that the LDMEx-FDM scheme with EISP can improve the reception performance of the fixed service without lowering the SNR threshold of the mobile service compared with the traditional LDM scheme in the ATSC 3.0 system. When the puncturing rate in the LDM-Ex-FDM scheme is configured as $1 / 12$, the performance of the fixed service is improved by $1.8 \mathrm{~dB}$, while the BER performance of the mobile service is not affected and the transmission rate is only reduced by $0.27 \mathrm{Mbps}$. In view of the $L D M$ symbols with overlapping constellations, our proposed LDM-Ex-FDM scheme with NEISP can increase the performance of the mobile service and fixed service by $0.9 \mathrm{~dB}$ and $1.05 \mathrm{~dB}$, respectively. Thus, since the SNR threshold of the $E L$ service is improved, many $E L$ holes can be avoided, thereby improving the overall coverage effect. In addition, to facilitate the implementation of the scheme, a low complexity DIC receiver is proposed accordingly. Simulation results show that, compared to the traditional SIC scheme, when the injection level is greater than $10 \mathrm{~dB}$, the BER performance of the proposed DIC scheme has almost no performance loss, and the complexity is significantly reduced.

It is worth pointing out that although the transmission rate of the mobile service is appropriately reduced, when the $C L$ configuration is robust, that is, the constellation is QPSK and the LDPC code rate is $5 / 15$, the CL can still fully meet the transmission of $720 \mathrm{p}$ mobile service in the typical scenario. Therefore, for the proposed LDM-Ex-FDM scheme, a reasonable compromise can be achieved between the performance of the $E L$ demodulation and the $C L$ transmission rate. For further research, we will study the 
optimization of the DIC scheme in the case that the $L D M$ power injection level is low and high-order modulation is adopted for the $C L$ service.

Author Contributions: Conceptualization, X.D. and M.L.; methodology, X.D., X.B., M.L.; software, X.D.; formal analysis, X.D.; investigation, X.D., X.B., M.L.; resources, X.D.; data curation, X.D.; project administration, X.D.; writing-original draft preparation, X.D., X.B., M.L.; writing-review and editing, X.D.; supervision, M.L. All authors have read and agreed to the published version of the manuscript.

Funding: This work was supported in part by the National Key R\&D Program of China under Grant 2019YFB1802703, in part by the Shanghai Excellent Academic Leader Program of China under Grant 18XD1404100, and in part by the Shanghai Technical Standard Project of China under Grant 18DZ2203900.

Institutional Review Board Statement: Not applicable.

Informed Consent Statement: Not applicable.

Data Availability Statement: Not applicable.

Conflicts of Interest: The authors declare no conflict of interest.

\section{References}

1. Xue, Y.; Yang, H.; Pan, C.; Song, J. Field trial of UHDTV over Digital Television Terrestrial Broadcasting network. In Proceedings of the 2019 IEEE International Symposium on Broadband Multimedia Systems and Broadcasting (BMSB), Jeju, Korea, 5-7 June 2019; pp. 1-3.

2. Eizmendi, I.; Velez, M.; Gómez-Barquero, D.; Morgade, J.; Baena-Lecuyer, V.; Slimani, M.; Zoellner, J. DVB-T2: The Second Generation of Terrestrial Digital Video Broadcasting System. IEEE Trans. Broadcast. 2014, 60, 258-271. [CrossRef]

3. Pan, C.; Zhang, C.; Yang, H.; Wang, J.; Li, X. Results of the DTMB-A Field Trials in Hong Kong. In Proceedings of the 2019 International Conference on Engineering and Telecommunication (EnT), Dolgoprudny, Russia, 20-21 November 2019; pp. 1-4.

4. Chernock, R.; Whitaker, J.C.; Wu, Y. ATSC 3.0-The Next Step in the Evolution of Digital Television. IEEE Trans. Broadcast. 2017, 63, 166-169. [CrossRef]

5. Song, J.; Zhang, C.; Peng, K.; Wang, J.; Pan, C.; Yang, F.; Wang, J.; Yang, H.; Xue, Y.; Zhang, Y.; et al. Key Technologies and Measurements for DTMB-A System. IEEE Trans. Broadcast. 2019, 65, 53-64. [CrossRef]

6. Jeon, S.; Kim, S.; Kim, J.; Yim, Z.; Seo, J. Field trial results of 4K-UHD over DVB-T2 single frequency network in Republic of Korea. In Proceedings of the 2016 IEEE International Symposium on Broadband Multimedia Systems and Broadcasting (BMSB), Nara, Japan, 1-3 June 2016; pp. 1-4.

7. Zhang, L.; Wu, Y.; Li, W.; Salehian, K.; Lafleche, S.; Wang, X.; Park, S.I.; Kim, H.M.; Lee, J.; Hur, N.; et al. Layered-Division Multiplexing: An Enabling Technology for Multicast/Broadcast Service Delivery in 5G. IEEE Commun. Mag. 2018, 56, 82-90. [CrossRef]

8. Zhang, L.; Li, W.; Wu, Y.; Xue, Y.; Sousa, E.; Park, S.; Lee, J.; Hur, N.; Kim, H. Using Non-Orthogonal Multiplexing in 5G-MBMS to Achieve Broadband-Broadcast Convergence With High Spectral Efficiency. IEEE Trans. Broadcast. 2020, 66, 490-502. [CrossRef]

9. Zhang, L.; Wu, Y.; Li, W.; Park, S.; Lee, J.; Hur, N.; Kim, H. Using Layered-Division-Multiplexing to Achieve Enhanced Spectral Efficiency in 5G-MBMS. In Proceedings of the 2019 IEEE International Symposium on Broadband Multimedia Systems and Broadcasting (BMSB), Jeju, Korea, 5-7 June 2019; pp. 1-7.

10. Islam, M.S.; Patwary, M.; Tait, R.; Peytchev, E. Layer division multiplexing for 5G DL transmission within ultra-dense heterogeneous networks. In Proceedings of the 2020 IEEE 91st Vehicular Technology Conference (VTC2020-Spring), Antwerp, Belgium, 25-28 May 2020; pp. 1-7.

11. Regueiro, C.; Montalban, J.; Barrueco, J.; Velez, M.; Angueira, P.; Wu, Y.; Zhang, L.; Park, S.; Lee, J.; Kim, H.M. LDM Core Services Performance in ATSC 3.0. IEEE Trans. Broadcast. 2016, 62, 244-252. [CrossRef]

12. Kim, H.J.; Kwon, S.; Kim, H.; Bae, J.; Hur, N. Performance Analysis of LDM and TDM systems for Three PLPs in DVB-T2. In Proceedings of the 2020 International Conference on Electronics, Information, and Communication (ICEIC), Barcelona, Spain, 19-22 January 2020; pp. 1-3.

13. Ahn, S.; Park, S.; Lee, J.; Kwon, S.; Liml, B.; Kim, H.M.; Hur, N.; Wu, Y.; Zhang, L.; Li, W.; et al. Performance Evaluation of ATSC 3.0 Mobile Service with LDM/TDM Under TU-6 Channel. In Proceedings of the 2018 IEEE International Symposium on Broadband Multimedia Systems and Broadcasting (BMSB), Valencia, Spain, 6-8 June 2018; pp. 1-9.

14. Park, S.; Lee, J.; Lim, B.; Kwon, S.; Seo, J.; Kim, H.M.; Hur, N.; Kim, J. Field Comparison Tests of LDM and TDM in ATSC 3.0. IEEE Trans. Broadcast. 2018, 64, 637-647. [CrossRef]

15. Lee, J.; Park, S.; Kwon, S.; Lim, B.; Kim, H.M.; Montalbán, J.; Angueira, P.; Zhang, L.; Li, W.; Wu, Y.-Y.; et al. Multiple Service Configurations Based on Layered Division Multiplexing. IEEE Trans. Broadcast. 2017, 63, 267-274. [CrossRef] 
16. Zhang, L.; Li, W.; Wu, Y.; Salehian, K.; Laflèche, S.; Hong, Z.; Park, S.; Kim, H.M.; Lee, J.; Hur, N.; et al. Using Layered-DivisionMultiplexing to Deliver Multi-Layer Mobile Services in ATSC 3.0. IEEE Trans. Broadcast. 2018, 65, 40-52. [CrossRef]

17. Kim, H.; Kim, J.; Park, S.I.; Lee, J.; Kwon, S.; Hur, N. Capacity Analysis and Improvement of LDM-Based Multiple-PLP Configurations in ATSC 3.0. IEEE Trans. Broadcast. 2021, 1-13. [CrossRef]

18. Kim, H.; Kim, J.; Park, S.I.; Lee, J.; Hur, N. Capacity Analysis for LDM-Based Multiple-PLP Configurations in ATSC 3.0. In Proceedings of the 2019 IEEE International Symposium on Broadband Multimedia Systems and Broadcasting (BMSB), Jeju, Korea, 5-7 June 2019; pp. 1-5.

19. Caire, G.; Taricco, G.; Biglieri, E. Bit-interleaved coded modulation. IEEE Trans. Inf. Theory 1998, 44, 927-946. [CrossRef]

20. Jin, H.; Peng, K.; Song, J. Backward Compatible Multiservice Transmission Over the DTMB System. IEEE Trans. Broadcast. 2014, 60, 499-510. [CrossRef]

21. ATSC Standard: Physical Layer Protocol, Document A/322: 2020. January 2020. Available online: https://www.atsc.org/wpcontent/uploads/2020/01/A322-2020-Physical-Layer-Protocol.pdf (accessed on 27 March 2021). 